\title{
Ground-based CCD astrometry with wide field imagers ${ }^{\star} \star \star$ III. WFI@2.2m proper-motion catalog of the globular cluster $\omega$ Centauri
}

\author{
A. Bellini ${ }^{1,2}$, G. Piotto ${ }^{1}$, L. R. Bedin ${ }^{2}$, J. Anderson ${ }^{2}$, I. Platais ${ }^{3}$, Y. Momany ${ }^{4}$, A. Moretti ${ }^{4}$, \\ A. P. Milone ${ }^{1}$, and S. Ortolani ${ }^{1}$
}

1 Dipartimento di Astronomia, Università di Padova, Vicolo dell'Osservatorio 3, 35122 Padova, Italy e-mail: [andrea.bellini;giampaolo.piotto; alessia.moretti; antonino.milone;sergio.ortolani]@unipd.it

2 Space Telescope Science Institute, 3700 San Martin Drive, Baltimore, MD 21218, USA e-mail: [bellini; bedin; jayander]@stsci.edu

3 Dept. of Physics and Astronomy, The Johns Hopkins University, Baltimore, MD 21218, USA e-mail: imants@pha.jhu.edu

4 INAF - Osservatorio Astronomico di Padova, vicolo dell'Osservatorio 5, 35122 Padova, Italy e-mail: yazan.almomany@oapd.inaf.it

Received 29 August 2008 / Accepted 3 October 2008

ABSTRACT

\begin{abstract}
Context. $\omega$ Centauri is the most well studied Galactic Globular Cluster because of its numerous puzzling features: significant dispersion in metallicity, multiple populations, triple main-sequence, horizontal branch morphology, He-rich population(s), and extended star-formation history. Intensive spectroscopic follow-up observing campaigns targeting stars at different positions in the color-magnitude diagram promises to clarify some of these peculiarities.

Aims. To be able to target cluster members reliably during spectroscopic surveys and both spatial and radial distributions in the cluster outskirts without including field stars, a high quality proper-motion catalog of $\omega$ Cen and membership probability determination are required. The only available wide field proper-motion catalog of $\omega$ Cen is derived from photographic plates, and only for stars brighter than $B \sim 16$. Using ESO archive data, we create a new, CCD-based, proper-motion catalog for this cluster, extending to $B \sim 20$.

Methods. We used high precision astrometric software developed specifically for data acquired by WFI@2.2m telescope and presented in the first paper of this series. We demonstrated previously that a 7 mas astrometric precision level can be achieved with this telescope and camera for well exposed stars in a single exposure, assuming an empirical PSF and a local transformation approach in measuring star displacements.

Results. We achieved a good cluster-field separation with a temporal base-line of only four years. We corrected our photometry for sky-concentration effects. We provide calibrated photometry for $U B V R_{\mathrm{C}} I_{\mathrm{C}}$ wide-band data plus narrow-band filter data centered on $\mathrm{H}_{\alpha}$ for almost 360000 stars. We confirm that the $\omega$ Cen metal-poor and metal-rich components have the same proper motion, and demonstrate that the metal-intermediate component in addition exhibits the same mean motion as the other RGB stars. We provide membership probability determinations for published $\omega$ Cen variable star catalogs.

Conclusions. Our catalog extends the proper-motion measurements to fainter than the cluster turn-off luminosity, and covers a wide area $\left(\sim 33^{\prime} \times 33^{\prime}\right)$ around the center of $\omega$ Cen. Our catalog is electronically available to the astronomical community.
\end{abstract}

Key words. catalogs - astrometry - Galaxy: globular clusters: general - Galaxy: globular clusters: individual: $\omega$ Cen stars: population II - stars: Hertzsprung-Russell (HR) and C-M diagrams

\section{Introduction}

The globular cluster $\omega$ Centauri $(\omega$ Cen) is the most luminous and massive cluster in the Galaxy. Observational evidence collected over the years has indicated that $\omega$ Cen is also the most puzzling stellar system in terms of stellar content, structure, and kinematics. Probably the most well studied of its peculiarities is one related to its stellar metallicity distribution (Norris \& Bessell 1975, 1977; Freeman \& Rodgers 1975; Bessell \& Norris 1976; Butler et al. 1978; Norris \& Da Costa 1995; Suntzeff \& Kraft 1996; Norris et al. 1996). There is a significant dispersion in the iron abundance distribution of $\omega$ Cen, with a

\footnotetext{
* Based on archive observations with the MPI/ESO $2.2 \mathrm{~m}$ telescope, located at La Silla and Paranal Observatory, Chile.

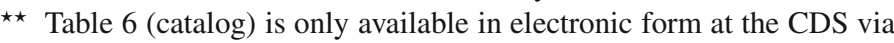
anonymous ftp to cdsarc.u-strasbg.fr $(130.79 .128 .5)$ or via http://cdsweb.u-strasbg.fr/cgi-bin/qcat?J/A+A/493/959
}

primary peak about $[\mathrm{Fe} / \mathrm{H}] \sim-1.7-1.8$ and a long tail, extending to $[\mathrm{Fe} / \mathrm{H}] \sim-0.6$, which contains another $3-4$ secondary peaks. It is possible to identify these metallicity peaks with distinct stellar populations (Pancino et al. 2000; Rey et al. 2004; Sollima et al. 2005; Villanova et al. 2007). Ground-based (Lee et al. 1999; Pancino et al. 2000) and Hubble Space Telescope (HST) (Anderson 1997; Bedin et al. 2004; Ferraro et al. 2004) photometry show clearly that $\omega$ Cen hosts different stellar populations. In particular, Pancino et al. (2000) demonstrated that the $\omega$ Cen red giant branch (RGB) consists of at least four distinct branches, spanning a wide range of metallicity. On the other hand, the $\omega$ Cen sub giant branch (SGB) has an intricate web of 5 distinct sequences, indicating an extended range of metallicity and age (see Bedin et al. 2004; Hilker et al. 2004; Sollima et al. 2005; Stanford et al. 2006; Villanova et al. 2007).

Anderson (1997), Bedin et al. (2004), and Villanova et al. (2007), by studying fainter stars with deep and high resolution 
HST photometry, demonstrated that the main sequence (MS) is divided into 3 distinct sequences. The spectroscopic study of the MS stars of $\omega$ Cen by Piotto et al. (2005) showed that the bluest of the main sequences is more metal rich than any of the redder sequences, which increased the ambiguity surrounding the cluster. An overabundance of $\mathrm{He}$ in the blue MS could reproduce the $\omega$ Cen MS photometric and spectroscopic properties (Bedin et al. 2004; Norris 2004; Piotto et al. 2005), although the origin of the puzzling MS morphology is still far from being understood.

A deeper insight into the enigmatic stellar populations of $\omega$ Cen should combine a deep, high resolution analysis of the inner and most crowded regions, with a wide field observations of the outskirts. While the first type of data has been provided adequately by $H S T$, wide field coverage of $\omega$ Cen requires groundbased data which are more difficult to obtain. Acquisition of data for a wider field-of-view will inevitably result in higher contamination by Galactic foreground/background populations. The only reasonable and efficient way to decontaminate the $\omega$ Cen outer stellar populations is by means of proper-motion analyses that help to isolate the Galactic contribution. The only available wide field $\omega$ Cen proper-motion catalog (van Leeuwen et al. 2000, hereafter vL00) is based on photographic observations and only provides measurements for stars brighter than $B \sim 16$. In this paper, we attempt to provide the first CCD-based proper motion catalog of $\omega$ Cen, extending the cleaned stellar populations down to $B \sim 20$, i.e. 4 mag deeper than vL00.

In the first paper of this series, Anderson et al. (2006), hereafter Paper I, demonstrated that WFI@2.2m observations, with a time base-line of only a few years, allow a successful separation of cluster members from Galactic field stars in the two GCs closest to the Sun: NGC 6121 and NGC 6397. In this paper, we apply the high precision astrometric and photometric techniques developed by Paper I to all available WFI@2.2m archive data of $\omega$ Cen.

In Sect. 2, we describe the available WFI observations of $\omega$ Cen, and the data sets that we used to derive proper motions. In Sect. 3, we discuss our photometric data reduction technique, the sky-concentration effect minimization, and the photometric calibration. In Sect. 4, we describe in detail how we treated the differential chromatic refraction (DCR) effects between the two epochs. Membership probability is discussed in Sect. 5, while in Sect. 6 we outline possible applications of our catalog. Finally, in Sect. 7, we summarize our results and describe the electronic catalog.

\section{Observations}

We used a collection of 279 archive images acquired between January 20, 1999 and April 14, 2003 at the ESO/MPI2.2m telescope at La Silla (Chile) equipped with the wide-field imager camera (WFI). A detailed log of observations is reported in Table 1. This camera, which consists of an array mosaic of $4 \times 2$ chips, $2141 \times 4128$ pixels each, has a total field of view of $34^{\prime} \times 33^{\prime}$, and a pixel scale of $0.238^{\prime \prime} /$ pixel. More details of the instrumental setup were given in Paper I. Images were obtained using $U, B, V, R_{\mathrm{C}}, I_{\mathrm{C}}$ wide-band and $658 \mathrm{~nm}\left(\mathrm{H}_{\alpha}\right)$ narrow-band filters, whose characteristics are summarized in Table 2.

For the derivation of proper motions, we used only $B$ and $V$ images acquired in April 1999 and April 2003 (see Sect. 4 for further details of this choice). The total field-of-view covered by the entire sample is indicated in Fig. 1, where axis coordinates are $(\Delta \alpha \cos \delta, \Delta \delta)$, expressed in units of arcmin from the $\omega$ Cen center (North is up, East is to the left). Concentric
Table 1. Description of the data set used for the WFI@2.2m catalog.

\begin{tabular}{|c|c|c|c|}
\hline Filter & $t_{\exp }$ & Seeing & Airmass \\
\hline \multicolumn{4}{|c|}{ January, 1999} \\
\hline$B_{842}$ & $3 \times 30 \mathrm{~s}, 1 \times 300 \mathrm{~s}$ & $1{ }^{\prime \prime} 0-1 . \prime 3$ & $\sim 1.20$ \\
\hline $658 \mathrm{~nm}$ & $1 \times 120 \mathrm{~s}, 5 \times 180 \mathrm{~s}, 1 \times 900 \mathrm{~s}$ & $1^{\prime \prime} 0-1 . .3$ & $1.15-1.18$ \\
\hline \multicolumn{4}{|c|}{ March, 1999} \\
\hline$V_{843}$ & $52 \times 200 \mathrm{~s}$ & $0 .{ }^{\prime} 8-1^{\prime \prime} .2$ & $1.1-1.2$ \\
\hline$I_{845}$ & $51 \times 150 \mathrm{~s}$ & $0.7-1^{\prime \prime} .5$ & $1.1-1.45$ \\
\hline \multicolumn{4}{|c|}{ April, 1999} \\
\hline$R_{844}$ & $1 \times 5 \mathrm{~s}, 1 \times 10 \mathrm{~s}, 1 \times 15,1 \times 30,5 \times 60 \mathrm{~s}$ & $1^{\prime \prime} 0-1 . .3$ & $1.3-1.6$ \\
\hline$I_{845}$ & $1 \times 5 \mathrm{~s}, 1 \times 10 \mathrm{~s}, 1 \times 20 \mathrm{~s}, 1 \times 45 \mathrm{~s}, 4 \times 90 \mathrm{~s}$ & $0.74-1 . .7$ & $1.4-1.9$ \\
\hline $658 \mathrm{~nm}$ & $2 \times 30,4 \times 120,5 \times 180 \mathrm{~s}$ & $0 .{ }^{\prime} 8-1^{\prime \prime} 15$ & $1.1-1.2$ \\
\hline \multicolumn{4}{|c|}{$($ epoch I) } \\
\hline$B_{842}$ & $1 \times 15 \mathrm{~s}, 1 \times 30 \mathrm{~s}, 1 \times 60 \mathrm{~s}, 5 \times 120 \mathrm{~s}$ & $0.75-1 . .3$ & $1.2-1.5$ \\
\hline$V_{843}$ & $3 \times 5 \mathrm{~s}, 3 \times 10 \mathrm{~s}, 1 \times 15 \mathrm{~s}, 2 \times 20 \mathrm{~s}$ & $0.7-1 .{ }^{\prime \prime} 0$ & $1.2-1.6$ \\
\hline & $1 \times 30 \mathrm{~s}, 4 \times 45 \mathrm{~s}, 10 \times 90 \mathrm{~s}$ & $0.76-1 . .36$ & $1.1-1.5$ \\
\hline \multicolumn{4}{|c|}{ July, 1999} \\
\hline$U_{877}$ & $2 \times 1800 \mathrm{~s}$ & $1 . .4-1 . .6$ & $\sim 1.14$ \\
\hline$B_{842}$ & $1 \times 10 \mathrm{~s}, 1 \times 30 \mathrm{~s}, 1 \times 40 \mathrm{~s}, 1 \times 300 \mathrm{~s}$ & $1 . .4-1{ }^{\prime \prime} 8$ & $1.14-1.25$ \\
\hline$V_{843}$ & $1 \times 10 \mathrm{~s}, 1 \times 20 \mathrm{~s}, 1 \times 150 \mathrm{~s}, 1 \times 240 \mathrm{~s}$ & $1 . " 3-1^{\prime \prime} 6$ & $1.15-1.25$ \\
\hline$I_{845}$ & $1 \times 10 \mathrm{~s}, 2 \times 20 \mathrm{~s}, 1 \times 240 \mathrm{~s}$ & $1^{\prime \prime} 13-1^{\prime \prime} 6$ & $1.17-1.25$ \\
\hline $658 \mathrm{~nm}$ & $3 \times 120 \mathrm{~s}, 1 \times 1200 \mathrm{~s}$ & $1^{\prime \prime} 13-1^{\prime \prime} .5$ & $1.18-1.23$ \\
\hline \multicolumn{4}{|c|}{ February, 2000} \\
\hline$B_{842}$ & $2 \times 30 \mathrm{~s}, 2 \times 240 \mathrm{~s}$ & $1^{\prime \prime} 45-1 . .7$ & $\sim 1.13$ \\
\hline$V_{843}$ & $1 \times 30 \mathrm{~s}, 2 \times 240 \mathrm{~s}$ & $1{ }^{\prime \prime} 1-11^{\prime \prime} 2$ & $\sim 1.13$ \\
\hline$I_{845}$ & $1 \times 30 \mathrm{~s}, 2 \times 240 \mathrm{~s}$ & $\sim 1^{\prime \prime} 0$ & $\sim 1.13$ \\
\hline \multicolumn{4}{|c|}{ March, 2000} \\
\hline$U_{841}$ & $4 \times 300 \mathrm{~s}, 1 \times 2400 \mathrm{~s}$ & $1^{\prime \prime} 1-11^{\prime \prime} 2$ & $1.17-1.38$ \\
\hline$V_{843}$ & $5 \times 30 \mathrm{~s}$ & $0.9-1^{\prime \prime} 4$ & $\sim 1.45$ \\
\hline \multicolumn{4}{|c|}{ June, 2002} \\
\hline$U_{877}$ & $4 \times 30 \mathrm{~s}, 7 \times 300 \mathrm{~s}$ & $0 .{ }^{\prime} 8-2^{\prime \prime} 0$ & $1.14-1.18$ \\
\hline$B_{878}$ & $1 \times 5 \mathrm{~s}, 3 \times 8 \mathrm{~s}, 9 \times 60 \mathrm{~s}$ & $0 .{ }^{\prime} 8-1^{\prime \prime} .5$ & $1.13-1.16$ \\
\hline$V_{843}$ & $3 \times 5 \mathrm{~s}, 3 \times 40 \mathrm{~s}, 3 \times 60 \mathrm{~s}$ & $0.75-2 .^{\prime \prime} 0$ & $\sim 1.13$ \\
\hline$I_{845}$ & $3 \times 20 \mathrm{~s}, 3 \times 40 \mathrm{~s}$ & $0.7-1^{\prime \prime} 4$ & $\sim 1.13$ \\
\hline \multicolumn{4}{|c|}{ April, 2003 (epoch II) } \\
\hline$B_{878}$ & $7 \times 40 \mathrm{~s} ; 7 \times 120 \mathrm{~s}$ & $0 .{ }^{\prime} 7-0 .{ }^{\prime} 9$ & $1.14-1.16$ \\
\hline$V_{843}$ & $7 \times 40 s ; 7 \times 120 s$ & $0 .{ }^{\prime} 8-1^{\prime \prime} 0$ & 1.13 \\
\hline
\end{tabular}

circles have diameters, if not specified, of $10^{\prime}$ (inner circle) and $30^{\prime}$ (outer circle), and are centered on the cluster center: $\alpha=201^{\circ} .69065, \delta=-47.47855$ (Van de Ven et al. 2006).

The first eight plots show the covered areas sorted by month and year, while in the bottom part of Fig. 1 the total coverage of all of the 279 images (on the left), and a zoom of the central part of the cluster (on the right) are shown. In the catalog presented in this work, the proper motion measurements are available only within the field-of-view in common between the two epochs used (see Fig. 1).

\section{Photometry, astrometry, and calibration}

\subsection{Photometric reduction}

For the reduction of the WFI@2.2m photometric data, we used the software img2xym_WFI, a modified version of img2xym_WFC.09x10 (Anderson \& King 2003), which was written originally for HST images, adapted successfully to ground-based data, and described in detail in Paper I. We closely followed the prescription given in Paper I for the data reduction of WFI images. This includes standard operations with the pixel data, such as de-biasing, flat-fielding, and correction for cosmic rays hits.

At the basis of the star position and flux measurements, there is the fitting of the empirical point spread function (PSF). In our approach, the PSF is represented by a look-up table on a very fine grid. It is well known that the shape of the PSF changes with position in WFI@2.2m chips. This variability can be modeled by an array of PSFs across the chip. The img2xym_WFI software works in a fully-automated way to find appropriate stars to 
Table 2. Characteristics of the used filters (from WFI manual) $\lambda_{\mathrm{c}}=$ central wavelength, FWHM $=$ full width at half maximum, $\lambda_{\mathrm{p}}=$ transmission peak wavelength, $T_{\mathrm{p}}=$ transmission percentage at peak level. (*) LWP means long wave pass: in this case the cutoff limit is determined by the CCD quantum efficiency.

\begin{tabular}{|c|c|c|c|c|}
\hline \multicolumn{5}{|c|}{ Wide-band filters } \\
\hline Name & $\begin{array}{c}\lambda_{\mathrm{c}} \\
{[\mathrm{nm}]}\end{array}$ & $\begin{array}{c}F W H M \\
{[\mathrm{~nm}]}\end{array}$ & $\begin{array}{c}\lambda_{\mathrm{p}} \\
{[\mathrm{nm}]}\end{array}$ & $\begin{array}{c}T_{\mathrm{p}} \\
{[\%]}\end{array}$ \\
\hline$U_{877}(U / 50)$ & 340.4 & $\overline{73.2}$ & 350.3 & 82.35 \\
\hline$U_{841}(U / 38)$ & 363.7 & 38.3 & 362.5 & 51.6 \\
\hline$B_{878}(B / 123)$ & 451.1 & 133.5 & 502.5 & 88.5 \\
\hline$B_{842}(B 99)$ & 456.3 & 99.0 & 475.0 & 91.2 \\
\hline$V_{843}(V / 89)$ & 539.6 & 89.4 & 523.0 & 87.0 \\
\hline$R_{844}\left(R_{\mathrm{C}}\right)$ & 651.7 & 162.2 & 668.5 & 93.9 \\
\hline$I_{845}\left(I_{\mathrm{C}} / l w p\right)$ & 783.8 & LWP* & 1001.0 & 97.6 \\
\hline \multicolumn{5}{|c|}{ Narrow-band filters } \\
\hline Name & $\begin{array}{c}\lambda_{\mathrm{c}} \\
{[\mathrm{nm}]}\end{array}$ & $\begin{array}{c}F W H M \\
{[\mathrm{~nm}]}\end{array}$ & $\begin{array}{c}\lambda_{\mathrm{p}} \\
{[\mathrm{nm}]}\end{array}$ & $\begin{array}{c}T_{\mathrm{p}} \\
{[\%]}\end{array}$ \\
\hline $658 \mathrm{~nm}\left(\mathrm{H}_{\alpha}\right)$ & 658.8 & 10.3 & 504.0 & 90.7 \\
\hline
\end{tabular}

represent the PSF adequately. For practical purposes, the number of PSF stars per chip can vary between 1 and 15, depending on the richness of the star-field. An iterative process is designed to work from the brightest to the faintest stars and find their precise position and instrumental flux. A reasonably bright star can be measured with a precision of $\sim 0.03$ pixel ( $\sim 7$ mas) on a single exposure.

Another problem of the WFI@2.2m imager is a large geometric distortion in the focal plane that effectively changes the pixel scale across the field-of-view. There are different ways to map this geometric distortion. We adopted a $9 \times 17$ element look-up table of corrections for each chip, derived from multiple, optimally-dithered observations of the Galactic bulge in Baade's Window (Paper I). This look-up table provides the most accurate characterization of geometrical distortions available for the WFI@2.2m. At any given location on the detector, a bilinear interpolation between the four closest grid points on the lookup table provides the corrections for the target point. The derived look-up table may have a lower accuracy at the edges of a field, because of the way in which the self-calibration frames were dithered (see Paper I). An additional source of uncertainty is related to a possible instability distortions in the WFI@2.2m reported earlier. This prompted us to use the local transformation method to derive proper motions (see Sect. 4).

\subsection{Sky-concentration correction}

Once we obtained star positions and instrumental fluxes for all images, we had to minimize the so-called "sky-concentration" effect. The WFI@2.2m camera is affected significantly by this kind of light contamination (Manfroid \& Selman 2001), which is caused by spurious reflections of light at discontinuities in the optics and the subsequent redistribution of light in the focal plane. The insidiousness of the effect is due to the fact that this redistribution of light affects both the science and the flat-field exposures.

Star fluxes are calculated by considering a local sky value, and therefore may be a negligible effect. However, since sky contamination also affects flat images, if it is not corrected properly during pre-reduction procedures, the quantum efficiency of the central pixel will be artificially lower with respect to that of the corner pixel. Consequently, the luminosity of a star measured in the middle of the mosaic camera will be underestimated by

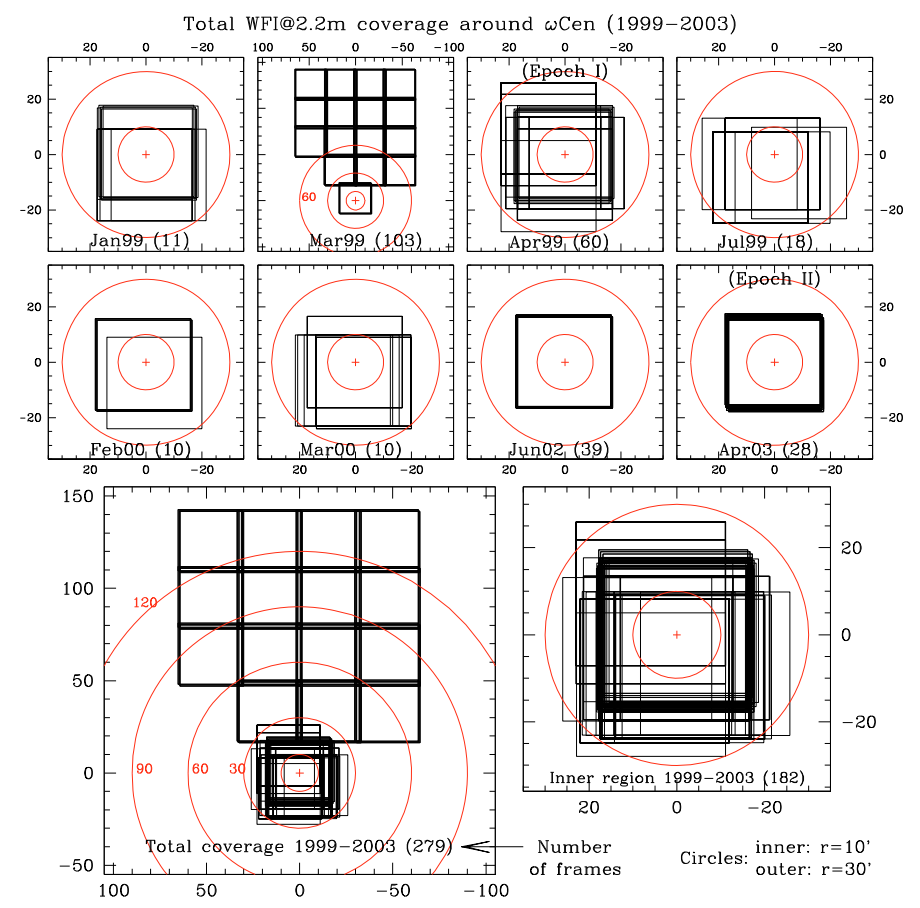

Fig. 1. Position footprint of the entire sample of WFI images around the $\omega$ Cen center (marked with a "+"). The first two rows show the covered areas sorted by month and year. In the lower panels of the figure, the total coverage of all of the 279 images (on the left), and a zoom-in of the central part of the cluster (on the right) are shown. The numbers in parenthesis after the dates represent the total number of images for that observing run. North is up, East to the left.

$\sim 0.1-0.2$ mag (in $V$ band) with respect to the luminosity of the same star detected close to the mosaic edges.

In Fig. 2a, we plot an instrumental ${ }^{1}$ color-magnitude diagram (CMD), which has been zoomed into the horizontalbranch (HB) region of $\omega$ Cen, obtained by combining all $V$ and $B_{878}$ images of April 2003. We chose this particular data set to highlight the effect of sky concentration on un dithered images. In fact, this data set has only one pointing, as shown in Fig. 1. The positions of stars on the CCD mosaic is almost identical from one image to another, implying a small contribution to the rms of the single star magnitude measurement due to sky-concentration effects. In Fig. 2a, we plotted 1252 stars with $\sigma_{V}<0.03 \mathrm{mag}$ and $\sigma_{B}<0.03 \mathrm{mag}$, where $\sigma_{V}$ and $\sigma_{B}$ are the standard errors of a single measurement (rms).

However, with only one pointing, sky concentration maximizes its effect on the relative photometry of stars located at different positions on the image. In Fig. 2a, we highlight the CMD of stars located at different positions on the CCD mosaic: with black dots (994 objects precisely), we show all stars between $2^{\prime}$ to $6^{\prime}$ from $\omega$ Cen center, which is close to the mosaic center ( $x=4150.69, y=4049.97$ on our master meta-chip). Red points are stars (258 objects) outside $12^{\prime}$. The displacement of the two HBs clearly shows that sky-concentration effects affect WFI photometry significantly if only one pointing is analyzed, and therefore needs to be corrected.

In our case, the analyzed data sets for different filters come from several pointings (except the case of $B_{878}$ images). In the process of matching all catalogs (for a given filter) to create a

\footnotetext{
1 Instrumental magnitudes are calculated to be $-2.5 \times \log \left(\Sigma_{i} D N_{i}\right)$, where $D N$ are the pixel's Digital Numbers above the local sky summed within a 10-pixel circular aperture. For a mean seeing of $0.8^{\prime \prime}$, saturation initiates at about $\sim-14.5$.
} 


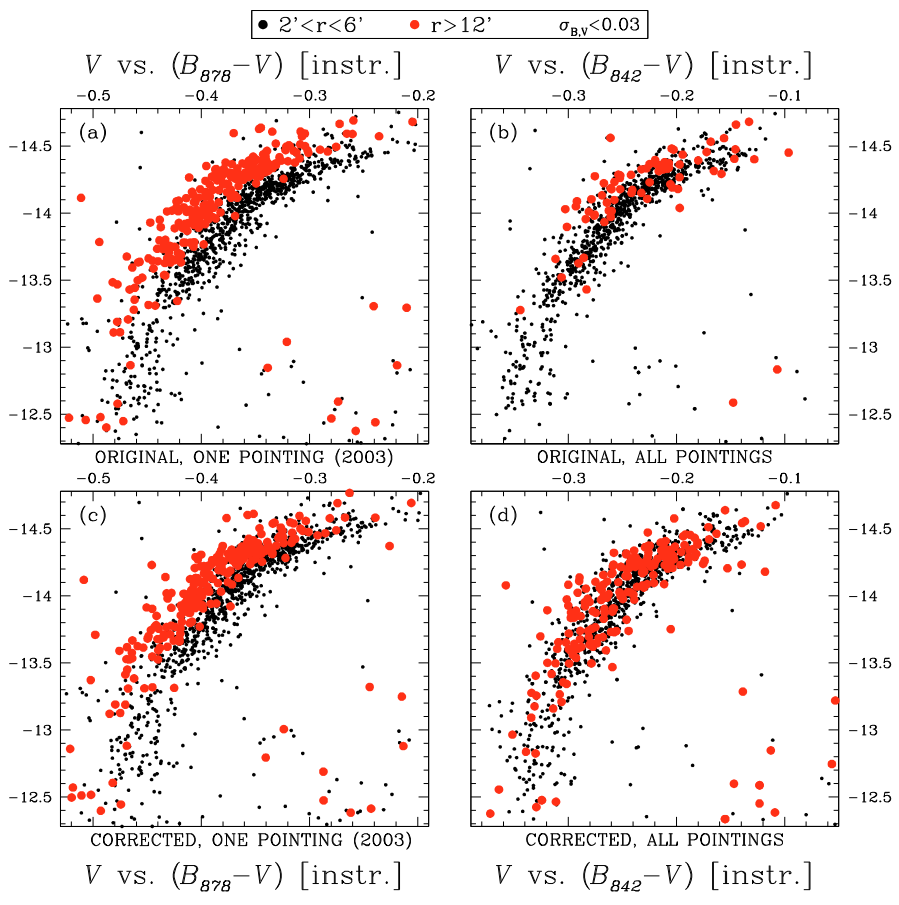

Fig. 2. CMDs zoomed into the HB region of $\omega$ Cen. All plotted stars have $\sigma_{B, V}<0.03$ mag. On the left, we used only images taken in April 2003 (i.e. only one pointing). On the right, we show photometry from all $V$ and all $B_{842}$ images that have independent pointings. Upper diagrams are for the original photometric catalogs. The lower ones are derived from sky-concentration-corrected catalogs. Black dots are stars located close to the mosaic center, while red dots are stars close to the mosaic edges.

single master-frame, the true sky-concentration effect is reduced. For a given star, we considered the mean of the star fluxes originating in different positions and for different pointings, so that the sky-concentration effect in the master frame was reduced. This process does, however, create systematic errors that affect the global photometry (see Selman 2001).

In Fig. 2b, we show the same zoomed HB region of $\omega$ Cen derived, in this case, by matching all the available $V$ and $B_{842}$ images obtained from the ESO archive. This data set contains several different pointings for both filters, so we were able to obtain photometry for the same stars located in some cases close to the mosaic center and in other pointings close to the mosaic edges. All plotted stars have again $\sigma_{B, V}<0.03 \mathrm{mag}$. In this case, only 972 stars (with the same previous convention, 896 black and 76 red) passed the selection criteria on the basis of photometric error. As explained before, matching catalogs for different pointing tends to minimize sky-concentration effects, but without an appropriate correction, rms of measurements for the stars are enhanced.

Andersen et al. (1995) studied the sky-concentration effect, typical of focal reducers, both by using simulations and analyzing data from the Danish telescope at La Silla. Their method for deriving the sky-concentration correction was based on the complex analysis of many star-field images taken at different orientations and positions during the night. Manfroid et al. (2001) applied a similar method to derive the sky-concentration effect for WFI@2.2m data, while Selman (2001) developed a method to estimate the sky-concentration effect by the analysis of the zeropoint variation in 3 dithered stellar frames, by evaluating this variations using a Chebyshev polynomial fit. They were able to reduce the internal error from 0.034 to 0.009 mag in the $V$ filter, and used the same polynomial fit to correct in addition the photometry in the other filters (see also Selman \& Melnick 2005). Koch et al. (2004) provided an analogous prescription to correct for the sky-concentration effect by comparing photometry derived with WFI and sloan digital sky survey (SDSS) data. Finally, Calamida et al. (2008) used some of the $\omega$ Cen images that we present in this work to correct for the positional effects of the WFI camera by means of photometric comparisons with the local standard stars of $\omega$ Cen.

The correction given by the ESO team, based on the $V$ filter, consists of a 9 th order bidimensional Chebyshev polynomial that should in principle be used also for the $U$ and $B$ filters. Selman (2001) found that his solution for the $V$ band was able to reduce the internal photometric error from 0.029 to $0.010 \mathrm{mag}$ in $B$, and from 0.040 to 0.014 in $U$, while for the other filters the $V$ correction failed to reduce the internal photometric error. Selman (2001) argued that this is probably due to problems associated with atmospheric variations affecting data for different filters. Unfortunately, by using the same polynomial coefficients to correct both $V$ and $B$ magnitudes, it is impossible to remove the color degeneracy due to the different response of the CCD to the sky concentration in the two different photometric bands. This degeneracy is of the order of $\sim 0.04-0.05$ mag in color in our $V$ versus $B-V$ CMD between inner stars $\left(r \sim 4^{\prime}\right)$ and outer stars $\left(r>12^{\prime}\right)$.

Our adopted solution consists of a self-consistent autocalibration of the sky-concentration map, and takes advantage of the high number of images analyzed, taken with different pointings. Below, we provide a description of the autocalibration procedure. We measured the raw magnitude $\operatorname{mag}_{i, j}$ of each $i$-star, in each $j$-image. We selected an image to be a reference frame (at the center of the dither pattern), and by using common stars with frame $j$ we were able to compute the average magnitude shifts to bring each image-catalog onto the magnitude reference frame $\left(\Delta_{j}\right)$.

If there were no systematic errors, the same stars measured at different positions in two different frames, should have the same magnitude value, within the random measurement errors:

$\operatorname{mag}_{i, 0}-\Delta_{0}=\ldots=\operatorname{mag}_{i, j}-\Delta_{j}=\ldots=\operatorname{mag}_{i, m}-\Delta_{m}$,

where $m$ is the total number of images in that filter, used to perform the autocalibration procedure.

However, the same star closer to the center of the camera is systematically fainter than when it is measured closer to the camera edges. For each star, measured in several different frames, and at different positions on the camera, we can compute an average of the values of the magnitudes in the reference system:

$\overline{\operatorname{mag}_{i}}=\left(\frac{1}{m}\right) \sum_{j=1}^{m}\left(\operatorname{mag}_{i, j}-\Delta_{j}\right)$.

In the same way, we can compute a residual for the $i$-star in the j-image:

$\delta_{i, j}=\left(\operatorname{mag}_{i, j}-\Delta_{j}\right)-\overline{\operatorname{mag}_{i}}$

All the residuals of stars close to the center will be systematically positive, and those close to the edges systematically negative. It appears clear that - at any given location on the camera - the average of the residuals from all the stars measured close to that location will provide a first spatial correction to our photometry. It also appears clear that the determination of the sky-concentration photometric correction will be an iterative process. 


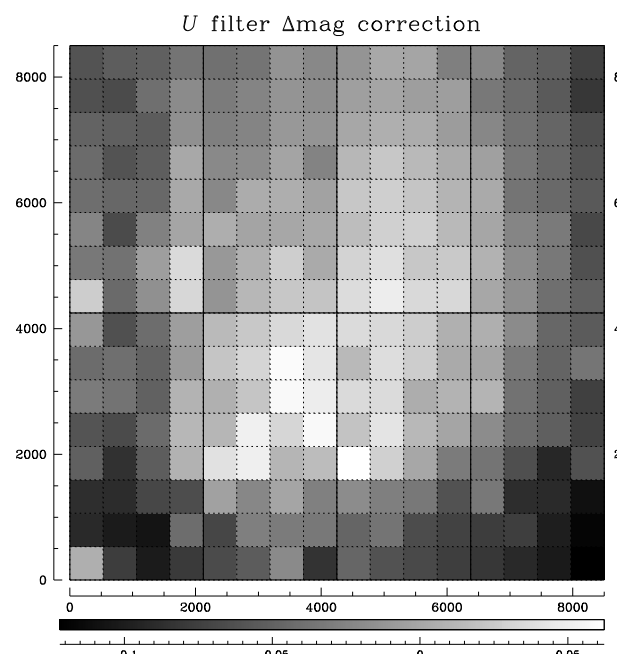

$R$ filter $\Delta$ mag correction

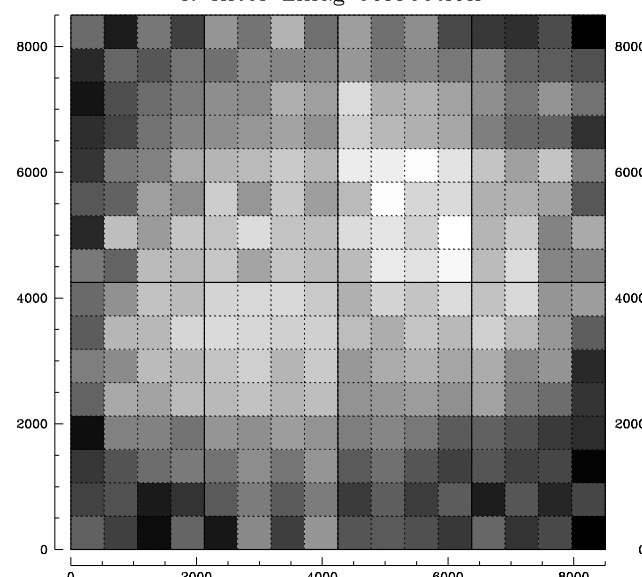

(1)

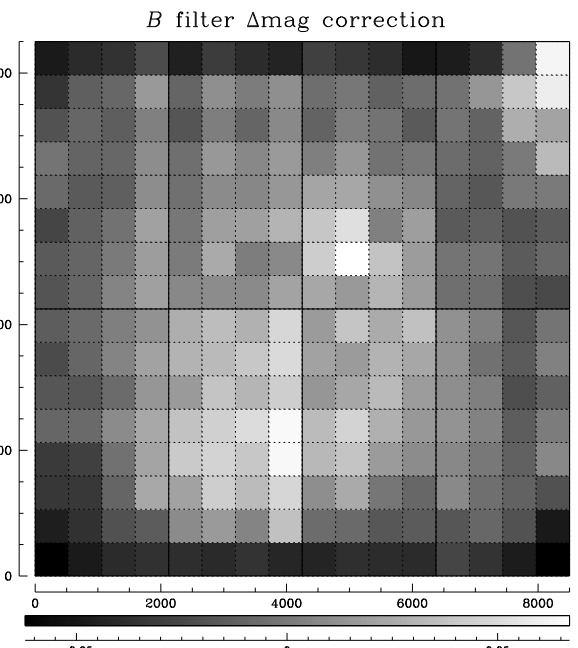
filter $\Delta$ mag correction

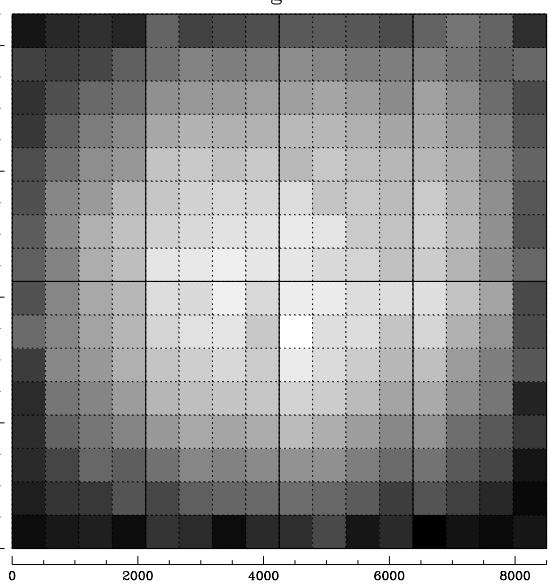

1000

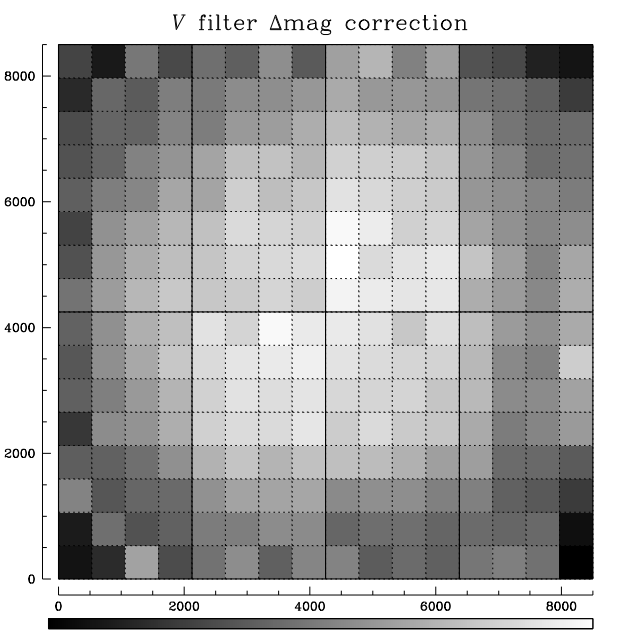

$H_{\alpha}^{-0.05}$ filter $\Delta$ mag correction

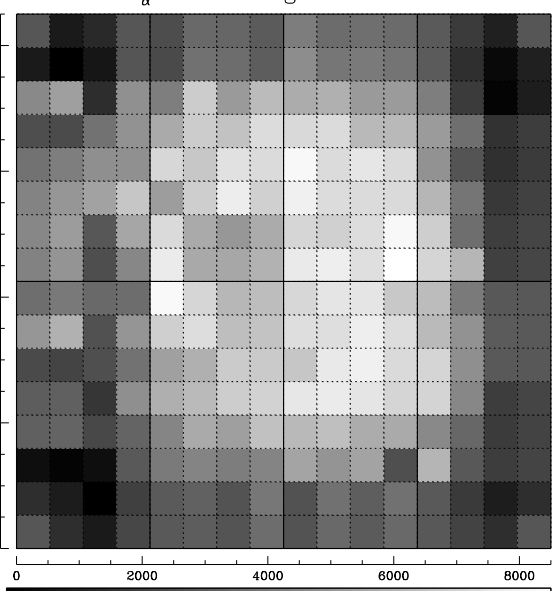

Fig. 3. Final $\Delta$ mag correction grids for the different filters ( $B$ means the $B_{842}$ filter only). Coordinates are in units of WFI pixels. Each WFI chip is highlighted by continuous black lines. Each element of the correction grids is colored according to the corresponding $\Delta$ mag correction applied. The grey scales are relative to the minimum/maximum correction for each filter.

To guarantee convergence, we applied half of the recommended correction at the given location, to all our image catalogs. We then recomputed the $\Delta_{j}$, and repeated the procedure until all the residual averages, at any given location, became smaller than $0.001 \mathrm{mag}$. The null hypothesis of this procedure is that the same star is imaged several times at different locations on the detector. To avoid systematic error, we also select, for each filter, the same number of exposures per different pointing (as much as possible with the existing database).

We use only those stars of the image catalogs with high S/N ratio (i.e. instrumental magnitude from -11 to -14 ) with a quality PSF-fit smaller than 0.1 (as defined by Anderson et al. 2008), and not too close to the cluster center (which does not necessarily coincide with the center of the camera) to avoid crowding, which compromises the photometric precision. The exact closeness to the center of the cluster depends on the image exposure time; we excluded stars within a radius of 1000-2000 pixels from the $\omega$ Cen center.

At this point, we define the expression of "given location". After several tests, we found a spatial grid of $16 \times 16$ boxes to be the most suitable compromise between a large number of residuals $\left(\delta_{i, j}\right)$ and a spatial resolution of correction sufficiently high to be useful. In Fig. 3, we show our final correction grids, respectively for $U, B_{842}, V, R_{\mathrm{C}}, I_{\mathrm{C}}$, and $\mathrm{H}_{\alpha}$ filters. For each filter, we present the final $16 \times 16$ element correction grid. Each element is colored according to the grey scale values (black for the minimum, white for the maximum). The grey scales vary linearly from the minimum to the maximum grid value for each filter. It is clear that sky concentration affects different filters in different ways, and each filter must therefore be corrected independently. To evaluate the correction at any point of the camera, we completed a bilinear interpolation of the closest 4 grid points. The adopted correction was less accurate close to the mosaic edges, where the peripheral grid-points have been stretched toward the boundaries. The available pointings for the other filters are also lower than for $B_{842}$ and $V$, implying a less effective correction of the sky concentration.

We emphasize that our correction is not completed by a starto-star comparison. After the spatial correction, a single star cannot have a lower random error (higher precision) than $\overline{\operatorname{mag}_{i}}$. However, the systematic errors (accuracy) of single stars relies on the quality of individual grid-point solutions, which were always calculated to be the average of residuals for several stars within each of the $16 \times 16$ cells. Even if our random errors for individual stars are $\sim 0.1 \mathrm{mag}$, with just 10 stars [in the worst case we still have at least 10 such stars] we can reduce our systematic errors to $\sim 0.03 \mathrm{mag}$. The condition that each star has to be observed in each of the $16 \times 16$ cells is the ideal case. Deviations from this ideal case occur frequently, although overall, we are close to achieving the optimal solution. 
The total amplitude of our correction for the $V$ filter is 0.13 mag. For the same filter, Manfroid \& Selman (2001) evaluated a 0.13 mag correction of similar spatial shape. Nevertheless, the two totally independent calibrations appear to be qualitatively the same. Based on the cell-to-cell scatter with the knowledge that sky-concentration is relatively flat, in $V$ and $I$ filters (for which we have more images) we estimate that the accuracy of our solution is as good as $\sim 0.03 \mathrm{mag}$. Although our corrections do not use any color information, we note that the postcorrected CMD is in excellent agreement with (to within a few hundreds of a magnitude) the HB location (Fig. 2).

To verify qualitatively the high quality of our skyconcentration correction procedure, we show in Fig. $2 \mathrm{~d}$ the same CMD region ( $V$ vs. $B_{842}-V$ ), derived using all the available images, after applying our correction. Stars located at different positions on the meta-chip are not affected by the sky-concentration effect, and are located in the same CMD region. The total number of plotted stars $\left(1227\right.$, all with $\sigma_{B, V}<0.03 \mathrm{mag}$ ) is comparable with that of Fig. 2a; this implies that we were able to remove the systematic contribution from our photometric rms values.

Our solution works well for the available $\omega$ Cen archive data sets (used to derive the corrections), but archive observations, in general, do not map every chip in a way that enables a skyconcentration correction that is universally applicable to be derived. We cannot guarantee that our solution can be applied to achieve the same positive results with other data sets. As proof of this issue, we applied our $B_{842}$ solution, to $B_{878}$ images with only one pointing.

Images collected using the $B_{842}$ and $B_{878}$ filters are not so different, in term of central wavelength (see Table 2): skyconcentration effects appear to be similar for almost identical filters (since they are related to atmospheric variations that affect the data for a range of different filters), so a photometric improvement is expected after correcting $B_{878}$ images with our $B_{842}$-derived solution. If our solution fails to correct the $B_{878}$ photometry, this is probably due to the different pointings of the two $B$ filters instead of the filters themselves. As shown in Fig. 2c, we found that a photometric improvement is present, with respect to Fig. 2a, but that the correction is not satisfactory.

\subsection{Instrumental UBVR $R_{C} I_{C}-H_{\alpha}$ photometric catalog}

We derived instrumental single-filter catalogs using all available images, by matching each chip individually to minimize the zeropoint differences between WFI chips. Included stars were measured in at least three distinct images. Photometric singlefilter catalogs were then linked to the astrometric one. Linked star positions agreed with those in the proper-motion catalog within 1 pixel for $B V R_{\mathrm{C}} I_{\mathrm{C}} H_{\alpha}$ filters, while for the $U$ filter we had to adopt a larger matching radius (3.5 pixels). This is mainly due to the poorer distortion solution in the $U$ band.

Due to the aforementioned sky-concentration minimization problem with $B_{878}$ images, our $B$ photometry refers to the $B_{842}$ filter.

As for the $U$ photometry, we used only $U_{877}$ images: in fact, $U_{841}$ is a "medium" rather than a wide-band filter, of quite different central wavelength and a low transmission efficiency with respect to $U_{877}$ (Table 2). If not specified otherwise, we refer to $B_{842}$ and $U_{877}$ simply using $B$ and $U$, respectively.

\subsection{Photometric calibration}

The photometric calibration of the WFI@2.2m data for $B V R_{\mathrm{C}} I_{\mathrm{C}}$ bands was performed using a set of $\sim 3000$ online wide field photometric $\omega$ Cen Secondary Standards stars (Stetson 2000, 2005). The Secondary Standards star catalog covers an area of about $30^{\prime} \times 30^{\prime}$ around the cluster center. We calibrated our $U$ instrumental photometry by cross-correlating our photometry with Momany et al. (2003) $U$ calibrated catalog (Stetson does not provide $U$ photometry for the $\omega$ Cen Secondary Standards). For $\mathrm{H}_{\alpha}$ calibration, we used as reference-standard stars the $3 \times 3$ central ACS/WFC mosaic photometric catalog in $F 658 \mathrm{~N}$ band (GO 9442), which was presented by Villanova et al. (2007). This HST catalog was obtained using img2xym_WFC.09x10 software; instrumental magnitudes were transformed onto the ACS Vega-mag flight system following Bedin et al. (2005), and by using the zero points of Sirianni et al. (2005).

For $B V R_{\mathrm{C}} I_{\mathrm{C}}$ bands, we matched our instrumental magnitudes and colors to the Stetson standard ones, and derived calibration equations by means of an iterative least squares fitting of a straight line (see Fig. 4). For these filters, we found that only a first-order dependency of the color term affects our instrumental magnitudes. The linearity of our calibration equations, which cover a wide range of colors (being derived from both HB and RGB stars) is evident from the plots of Fig. 4.

As in the calibration of our $U$ instrumental photometry, the Momany et al. (2003) catalog was not corrected for skyconcentration effects. We found a magnitude dependence related to the star positions. We adopted a straight line fit to derive the calibration equation, because we were unable to consider the different color/magnitude dependencies individually. Therefore, our calibrated $U$ magnitudes were not more reliable than the 0.15 mag level (maximal error).

For the $\mathrm{H}_{\alpha}$ filter, we again performed a straight line fit to derive the calibration equations, even though the data appear to suggest a second order color effect (see the corresponding panel of Fig. 4). ACS/WFC data cover only the inner $\sim 10^{\prime} \times 10^{\prime}$ region of our catalog, and are therefore taken in extremely crowded conditions. This effect might strongly influence our photometry mimicking the aforementioned second-order effect. In Fig. 5, we show in the left panels our photometric errors for each filter, as a function of the corresponding magnitude. The photometric errors (standard deviation) have been computed from multiple observations, all reduced to the common photometric reference frame in the chosen bandpass. In the right panels of Fig. 5, we plot the photometric standard error of the mean - to be defined as $\sigma / \sqrt{N-1}$, where $N$ is the total number of observations - versus the magnitude, for each filter.

To illustrate more clearly the dependence of our photometric rms on crowding, we show (for the $V$ filter only) in Fig. 6 the photometric rms $\sigma_{V}$ with respect to $V$ for stars within $7^{\prime}$ (top panel), from $7^{\prime}$ to $14^{\prime}$ (middle panel), and outside $14^{\prime}$ (bottom panel). Stars located in the most crowded region of the field suffer higher uncertainty in their photometry.

Our final catalog consists of about 360000 stars, in $U B V R_{\mathrm{C}} I_{\mathrm{C}}$ wide-band and $658 \mathrm{~nm}$ narrow-band filters, covering a wide area $\left(\sim 33^{\prime} \times 33^{\prime}\right)$ centered on $\omega$ Cen. We reach 3 mag in $V$ band below the TO point with a photometric rms of 0.03 mag.

\subsection{Zeropoint residuals}

Even if our sky-concentration correction works well, residuals are still present, especially close to the corners of our final catalog. Due to the wide field area analyzed in this work, there is also the possibility of a contribution from differential reddening. Using ubvy Strömgren and V I photometry, Calamida et al. (2005) developed an empirical method to estimate the 

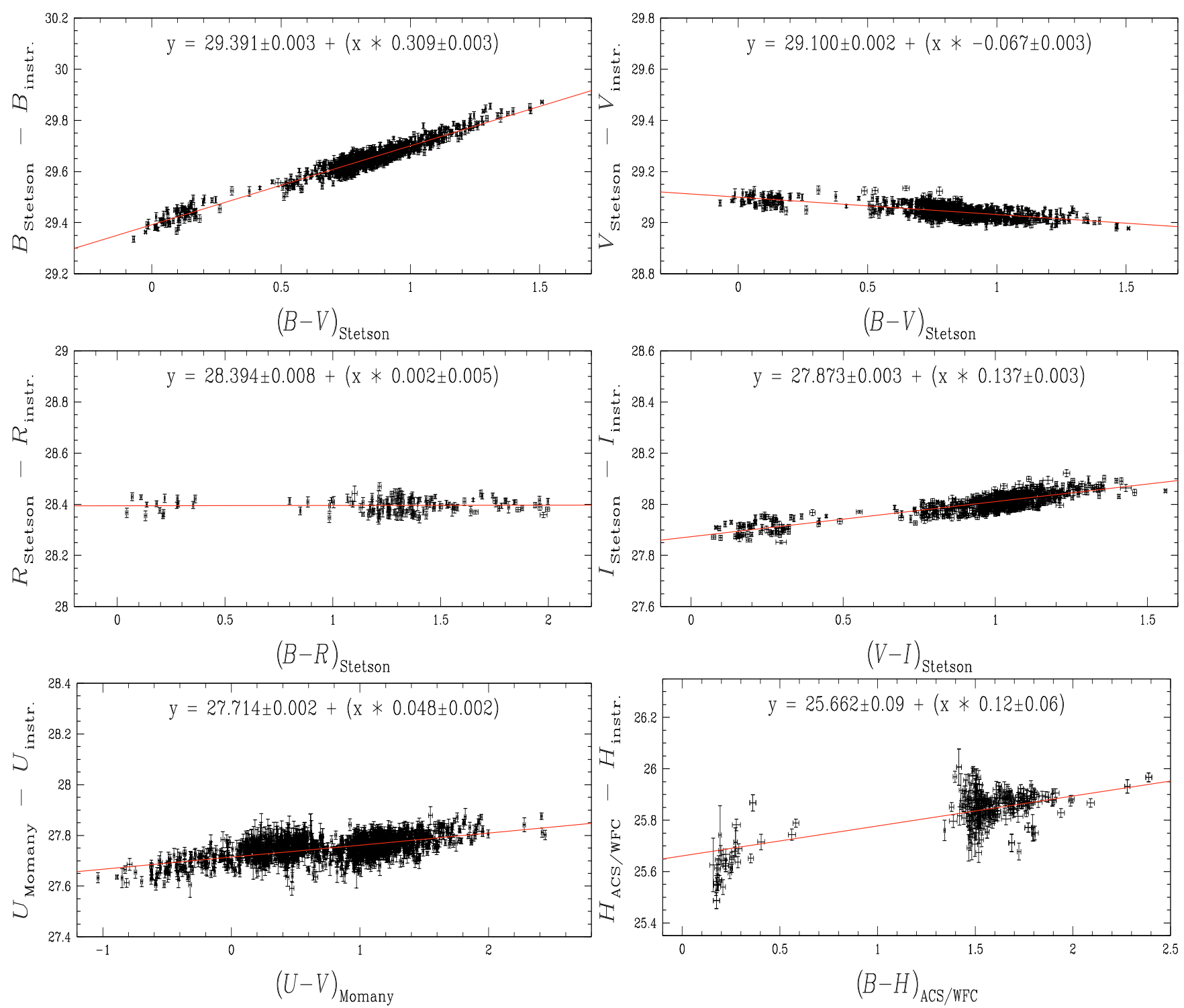

Fig. 4. These figures show our calibration fits with the adopted color equations. For the $B, V, R_{\mathrm{C}}, I_{\mathrm{C}}$ filters we used the on-line set of standard stars provided by Stetson, while for $U$ and $\mathrm{H}_{\alpha}$ bands we used as reference stars the Momany et al. (2003) catalog and the ACS/WFC catalog of Villanova et al. (2007), respectively. See text for more details.

differential reddening of $\omega$ Cen. The authors found that the reddening can vary in the range $0.03 \lesssim E(B-V) \lesssim 0.15$ from Strömgren filters, and $0.06 \lesssim E(B-V) \lesssim 0.13$ from $V I$ filters, within their analyzed field-of-view of $14^{\prime} \times 14^{\prime}$, which was centered on the center of $\omega$ Cen. However, the results by Calamida et al. (2005) were questioned by Villanova et al. (2007), and the quantitative value of the differential reddening still needs to be confirmed.

In the case of the $B-V$ color, the maximum zeropoint residual in our final catalog is less than $0.1 \mathrm{mag}$. To minimize any zeropoint variations, we used a method similar to that described by Sarajedini et al. (2007). Briefly, we defined the fiducial ridgeline of the most metal-poor component of the $\omega$ Cen RGB and tabulate, at a grid of points across the field, how the observed stars in the vicinity of each grid point may lie systematically to the red or the blue of the fiducial sequence; this systematic color offset is indicative of the local differential reddening.

Our online catalog magnitudes are not corrected for differential reddening to enable the user to adopt their preferred correction method in removing differential reddening and zeropoint residuals.

\section{Proper-motion measurements}

To complete the proper-motion analysis, we used only the $B$ and $V$ images taken in April 1999 (epoch I) and April 2003 (epoch II). This choice was due to the fact that: (i) we have a fine-tuned geometric distortion correction map for $V$ filter (Paper I), which has been proven to work well for the two $B$ filters (Paper I); (ii) it offers the widest possible time base-line of $\sim 4$ yrs; and (iii) we have a relatively high number of images in both epochs, and with relatively deep exposures.

We first photometrically selected probable cluster members in the $V$ versus $B-V$ color-magnitude diagram. These stars are located on the RGB (see the RGB selections in the top-panel of Fig. 13, within the magnitude interval $14.6<V<17.2$. We used these stars only as a local reference frame to transform the coordinates from one image to the system of the other images at different epochs and therefore derive relative proper motions. By using predominantly cluster stars, we ensure that proper motions will be measured relative to the bulk motion of cluster stars. The expected intrinsic velocity dispersion of $\omega$ Cen stars for which we can measure reliable proper motions, is between 10 

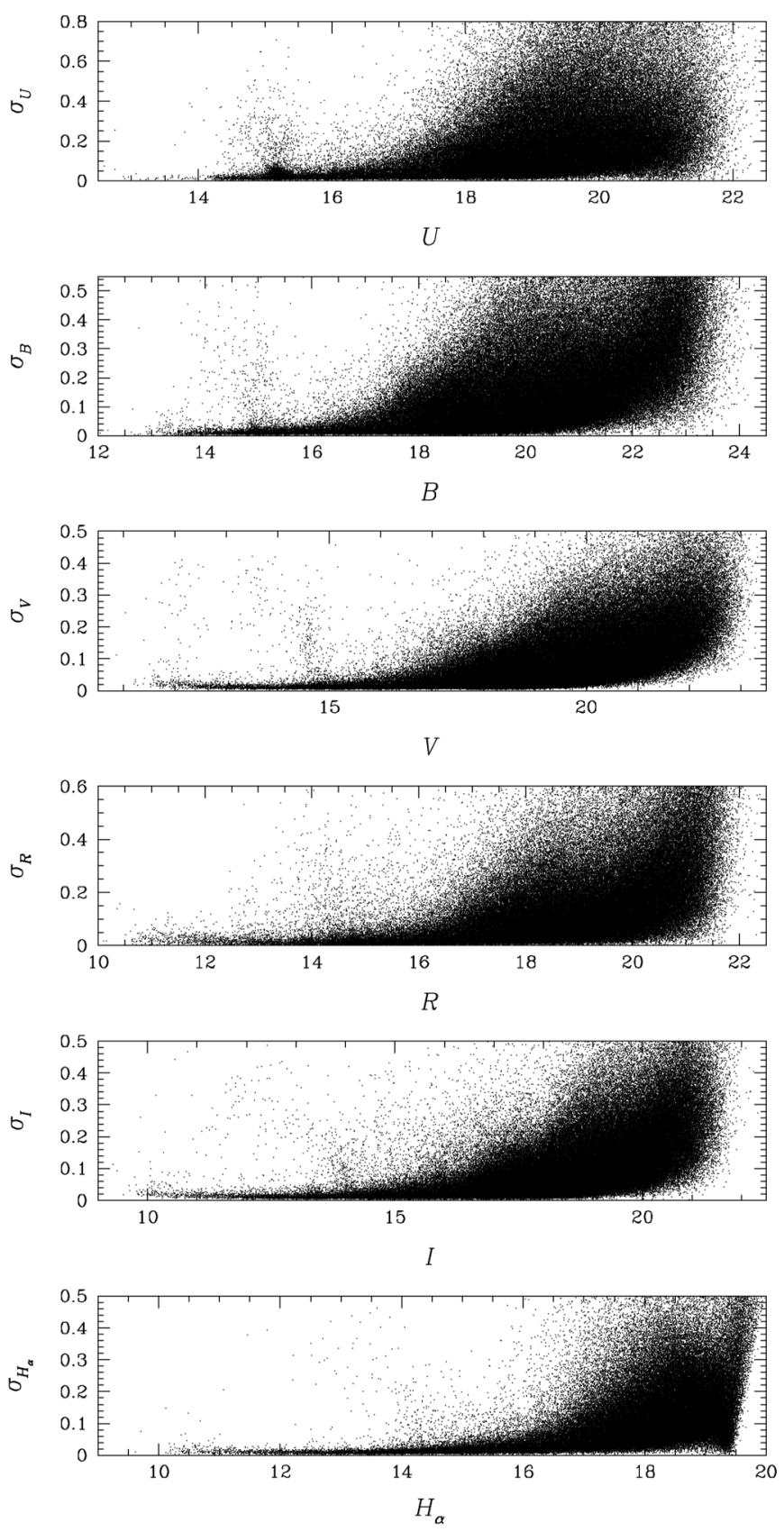
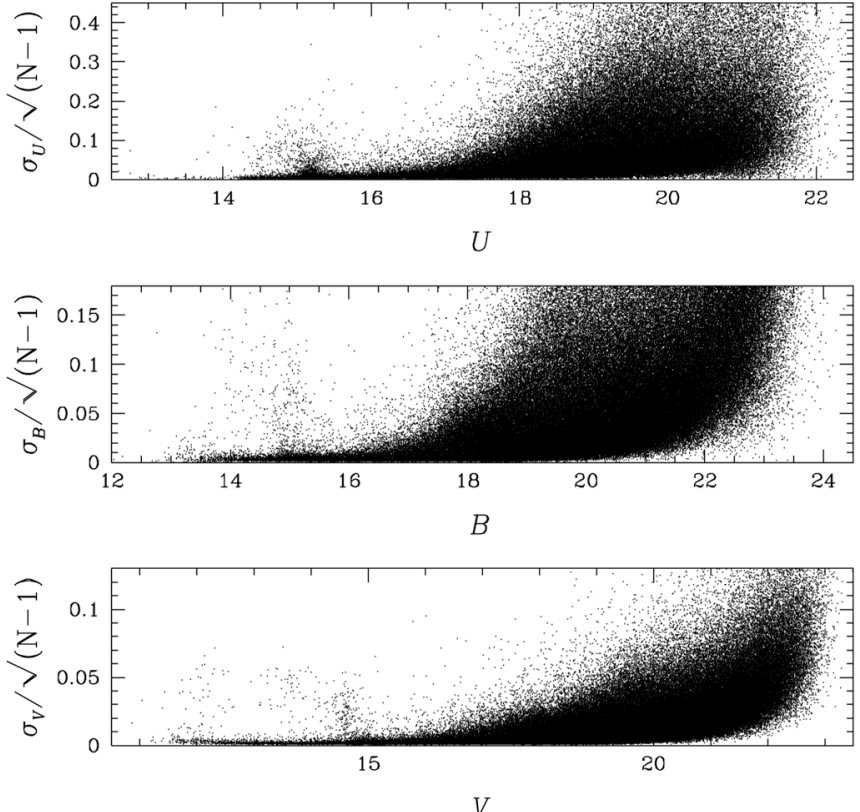

V
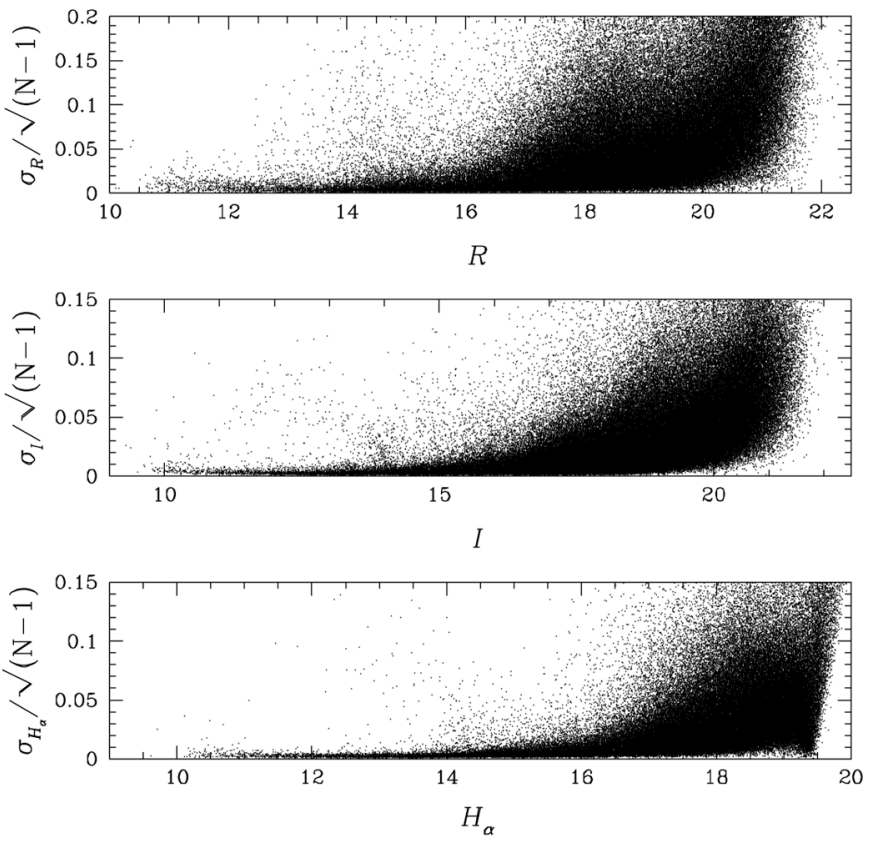

Fig. 5. Left panels: plot of magnitude rms $(\sigma)$ as a function of magnitude. Right panels: plot of the standard error of the mean $(\sigma / \sqrt{N-1}$, where $N$ is the number of measurements) as a function of magnitude.

and $15 \mathrm{~km} \mathrm{~s}^{-1}$ (Merritt et al. 1997). If we assume a distance of $5.5 \mathrm{kpc}$ for $\omega$ Cen, as reported by Del Principe et al. (2006), and isotropic distribution of stars (good to first order), then these

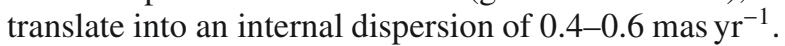

Over the four-year epoch, the difference would result in a displacement of only 1.5-2.3 mas, which is a factor of 3 smaller than the random measurement errors ( $\sim 7$ mas). Conversely, the tangential velocity dispersion of field stars is a factor of $\sim 10$ larger than the intrinsic velocity dispersion of the cluster. For field stars, proper motions are clearly not negligible with respect to measurement errors, and this has an adverse effect on the coordinate transformations. We removed iteratively stars from the preliminary photometric member list that had proper motions clearly inconsistent with cluster membership, even though their colors placed them close to the fiducial cluster sequence.
To minimize the effects of geometric-distortion-solution residuals on proper motions, we used local transformations $\sim 30$ arcsec. These were well-measured cluster stars of any magnitude selected to be on the same CCD chip, as long as their preliminary proper motion is consistent with cluster membership. No systematic errors larger than our random errors are visible close to the corners or edges of chips.

To avoid possible filter-dependent systematic errors, we measured proper motions in the $V$ and $B$ bandpasses only, for which the geometrical distortion corrections were derived originally (Paper I). Individual errors of proper motions for single stars were estimated as described in Sect. 7.3 of Paper I. For both epochs separately, we estimated the intra-epoch rms error from all same-epoch plates transformed locally to the same reference frame. The proper-motion errors were computed to be based on the closest 20 reference stars, typically extending over 

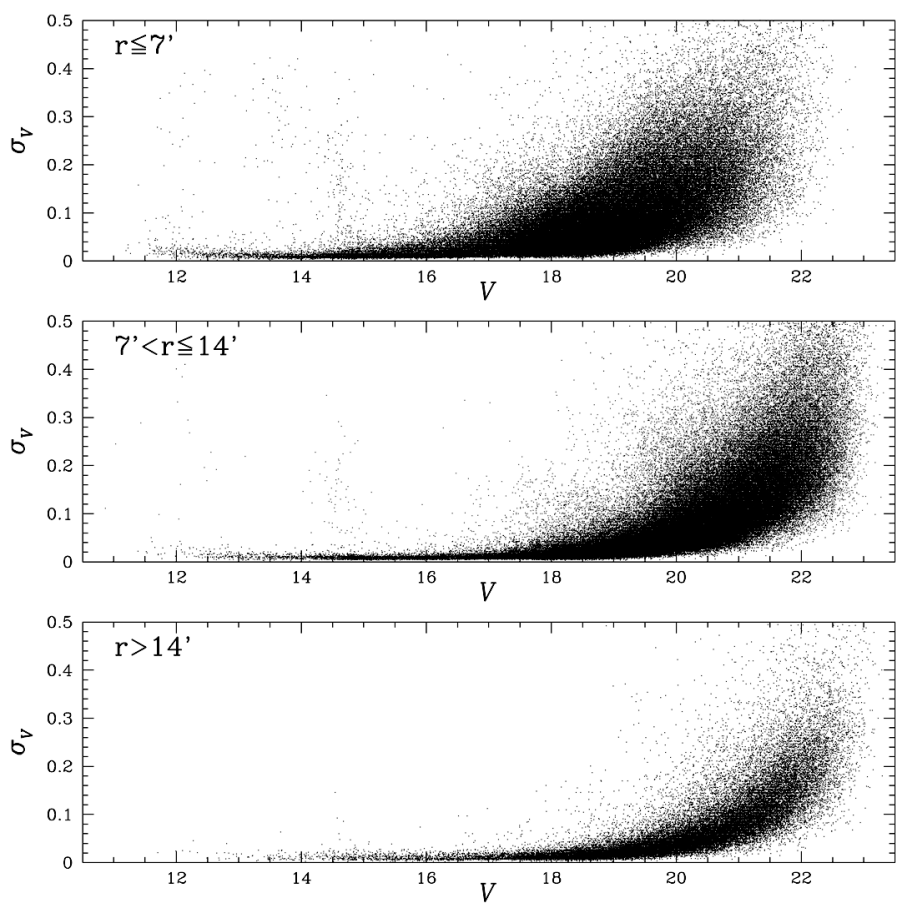

Fig. 6. Photometric rms for stars within $7^{\prime}$ (top panel), from $7^{\prime}$ to $14^{\prime}$ (middle panel), and outside 14' (bottom panel).

the rms of the proper motion, obtained by solving locally each first-epoch frame into each second-epoch frame. These errors, however, were not entirely independent because the same frames were used more than once. Therefore, to obtain our most reliable estimate of the proper-motion standard error, we added in quadrature the intra-epoch rms of each epoch.

In Fig. 7, we show our proper-motion rms, in units

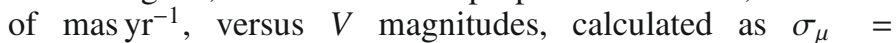
$\sqrt{\sigma_{\mu_{\alpha} \cos \delta}^{2}+\sigma_{\mu_{\delta}}^{2}}$. The top panel presents stars within $7^{\prime}$ of the center of $\omega$ Cen, the middle panel is for stars between $7^{\prime}$ and $14^{\prime}$, while the lower panel shows the errors for stars outside $14^{\prime}$. The vertical dashed line indicates the saturation limit of the deepest exposures $(V=14.6)$, while the continuous line is at $V=16.5$, the vL00 faintness limit. The precision of our proper-motion measurement is $\lesssim 0.03$ WFI pixels in 4 yrs down to $V \sim 18 \mathrm{mag}$

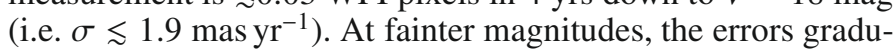

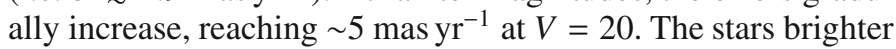
than $V \sim 13$ mag show a higher dispersion because of the image saturation even in the shortest exposures. Horizontal lines in Fig. 7 indicate the median proper-motion rms of unsaturated

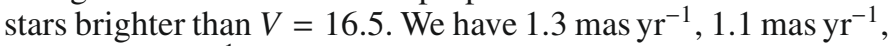

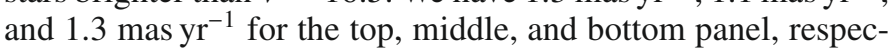
tively. The higher value for the inner stars is due to crowding while, for the outer stars, there is a combination of three factors:

(1) our geometrical distortion solution is less accurate close to the WFI mosaic edges;

(2) there are fewer cluster members, per unit area, usable as a reference for deriving proper motions;

(3) we have a lower number of images that overlap with the external areas of the field-of-view.

\subsection{Cluster CMD decontamination}

To probe the effectiveness of our proper motions in separating cluster stars from the field stars, we show in Fig. 8 the vectorpoint diagrams (VPDs, top panels), and the CMDs in the $V$ vs.
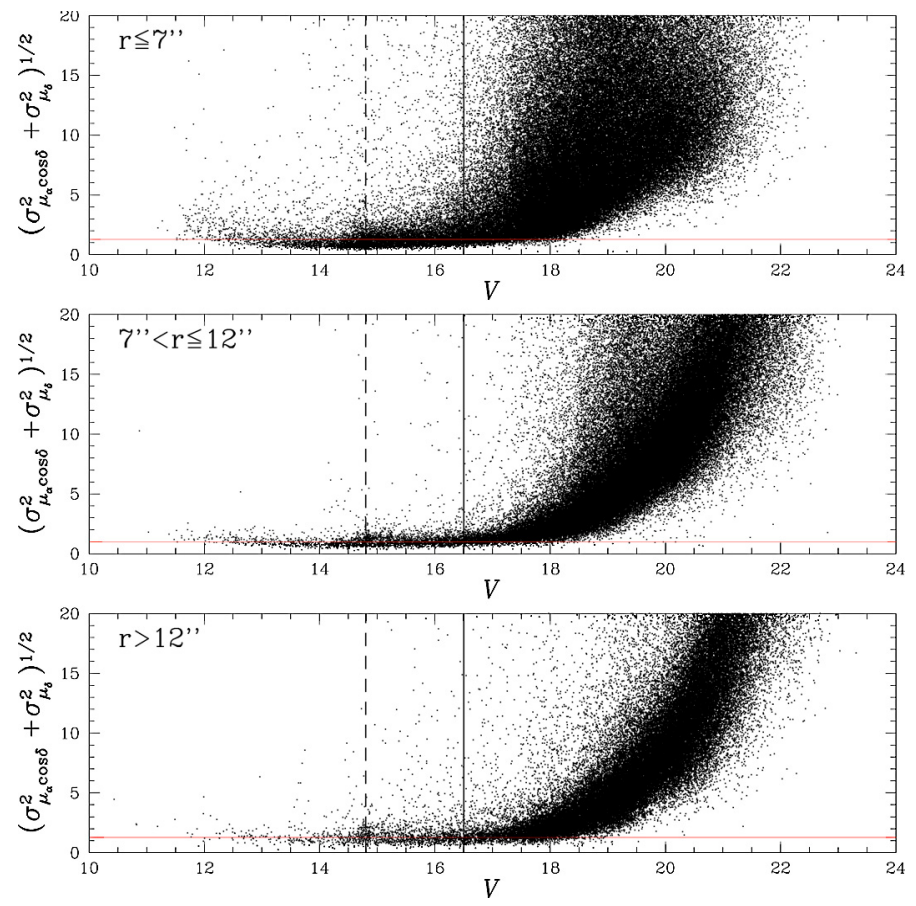

Fig. 7. Proper-motion errors for stars within $7^{\prime}$ (top panel), from $7^{\prime}$ to $14^{\prime}$ (middle panel), and outside 14' (bottom panel). The proper-motion errors are expressed in the units of mas $\mathrm{yr}^{-1}$.

$B-V$ plane (bottom panels). In the left panels, we show the entire sample of stars; the middle panels display what we considered to be probable cluster members; the right panels show predominantly the field stars. Plotted stars have a $V$ rms lower than 0.03 mag.

In the VPDs, we draw a circle around the cluster centroid of

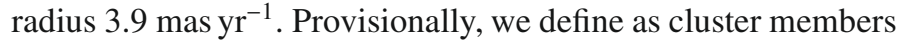
all points in the VPD within this circle. The chosen radius is the optimal compromise between missing cluster members with uncertain proper motions, and including field stars that have velocities equal to the cluster mean proper motion. Even this approximate separation between cluster and field stars demonstrates the power of proper motions derived in this study. A description of membership probability is given in Sect. 5 .

\subsection{Differential chromatic refraction (DCR)}

The DCR effect causes a shift in the photon positions in the $\mathrm{CCD}$, which is proportional to their wavelength, and a function of the zenithal distance: blue photons will occupy a position that differs from that of red photons. The DCR effect is easier to detect and remove from CCDs, due to their linearity.

Unfortunately, within each epoch, the available data sets are not optimized to perform the DCR correction directly (Monet et al. 1992), because the images have not been taken at independent zenithal distances. We can, however, check if possible differences in the DCR effect between the two epochs could generate an apparent proper motion for blue stars relative to red stars.

We selected four samples of stars located on the $\mathrm{HB}$, and five on the RGB, as shown in Fig. 9 (top panel), with different colors to estimate the DCR effect, in a magnitude interval of $1.8 \mathrm{mag}$ in $V(14.4 \leq V \leq 16.2)$, with proper motions $\leq 3.8$ mas yr $^{-1}$ and $\mathrm{rms} \leq 1.9 \mathrm{mas} \mathrm{yr}^{-1}$. We chose this magnitude range to: (i) avoid luminosity-dependent displacements (if any); (ii) include 


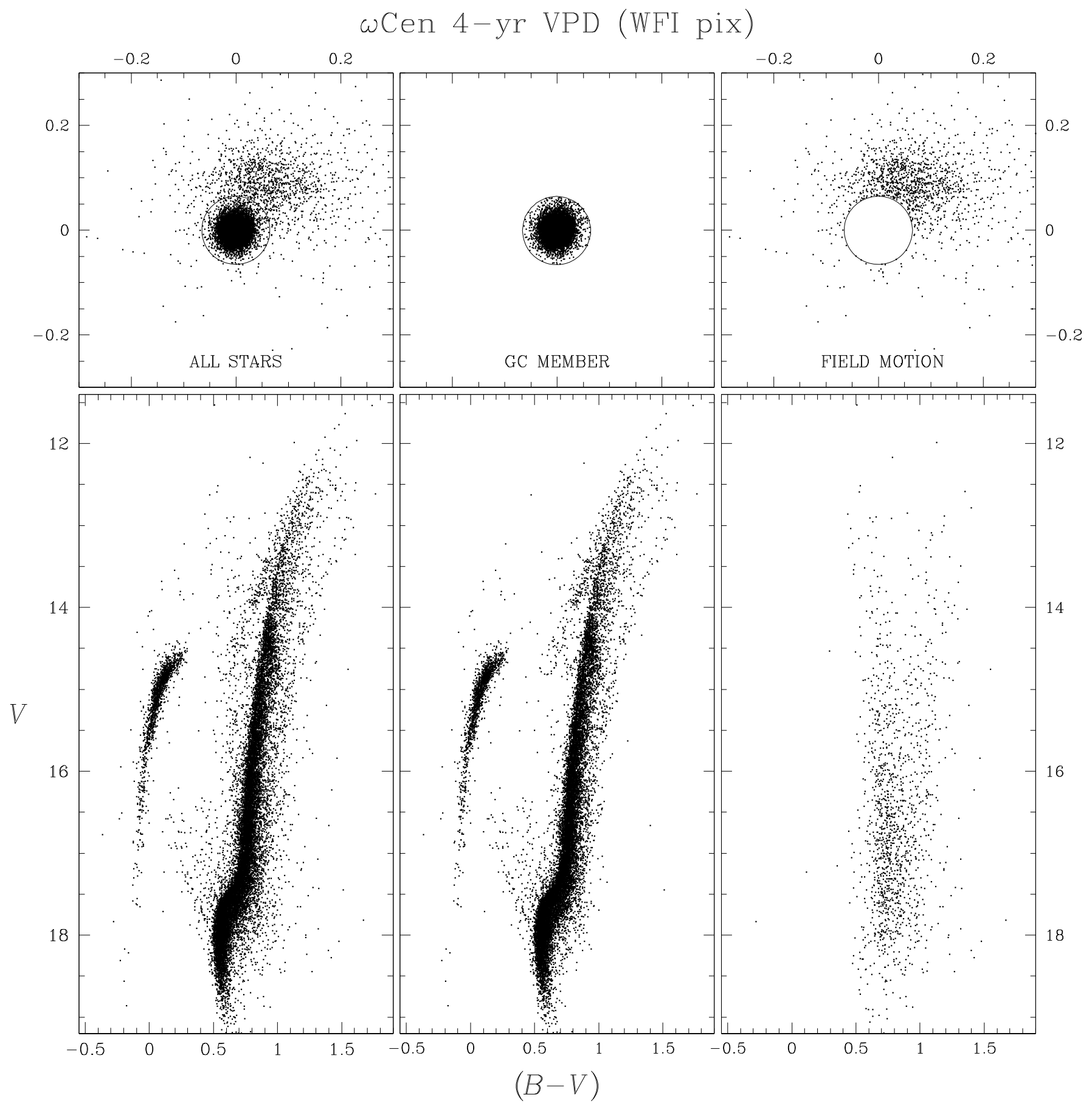

Fig. 8. Top-panels: proper-motion vector-point diagram. Zero point in VPD is the mean motion of cluster stars candidates. Bottom panels: calibrated $V,(B-V)$, color-magnitude diagram. Left: the entire sample. Center: stars in VPD with proper motion within 0.065 pixels (i.e.

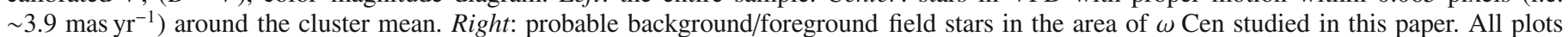

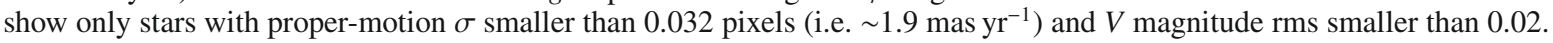

stars with a low rms in positions and fluxes (see Figs. 5, 7), excluding saturated stars; and (iii) cover the widest possible color baseline according to the above points (i) and (ii). For each of the nine samples, we derived the median color and the median proper motion along $\mu_{\alpha} \cos \delta$ and $\mu_{\delta}$, and their respective errors. Proper motions were expressed in the terms of a displacement over 4 years, that is in the units of WFI pixels along the $X$ and $Y$ axes of a detector (parallel to the RA and Dec directions).

In Fig. 9, we show, in the top panel, the selected stars on the CMD used to examine the DCR effect; the linear fits adopted for the $X$ and $Y$ displacements are shown in the lower part of the figure. We found a negligible DCR effect along both $X$ and $Y$ axes. For this reason, we have not corrected our measurements for this effect.

In Fig. 10, we show, on the left, our calibrated $V, B-V$ CMD, divided into eight magnitude bins. In each bin, we adopted different selection criteria to identify cluster members, which were more stringent for stars with more reliable measurements from data of high signal-to-noise ratio, and less restrictive for star with less precise measurements. Plotted stars have a proper-motion 

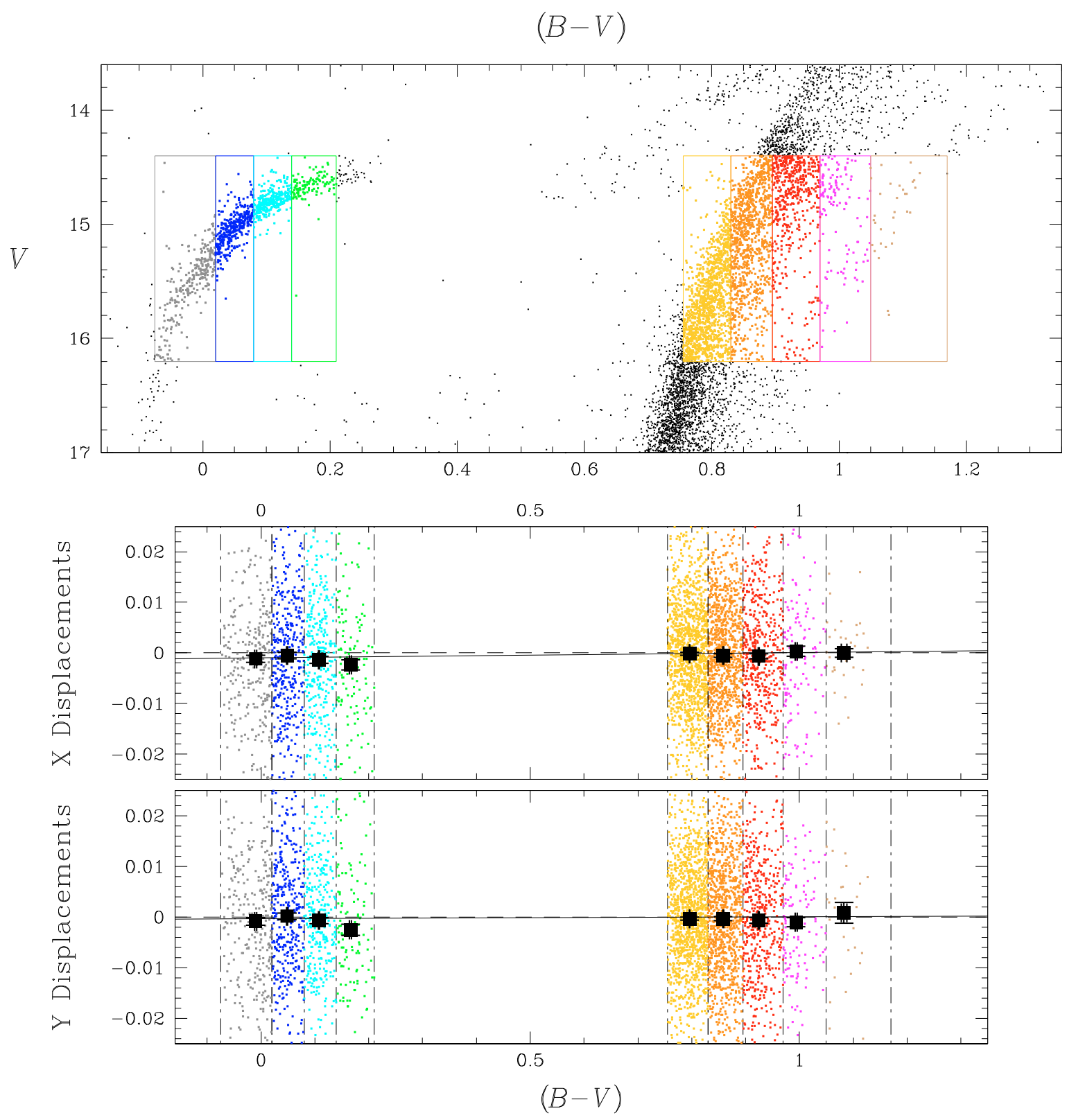

Fig. 9. Top panel: selected stars in the CMD of $\omega$ Cen for DCR effect correction. Middle and bottom panels: star displacements along $X$ and $Y$ axes, as a function of stars $(B-V)$ color. The median shift of the nine samples are also showed, with errors. The continuous lines show the adopted fits used to quantify the DCR effects.

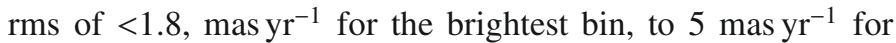
the faintest one. The Photometric rms for both the bands range between $0.02 \mathrm{mag}$ for the brightest bin to 0.05 for the faintest one, which is sufficient to include main-sequence stars down to $V=20$. For each magnitude bin, we considered as cluster members those stars with a proper motion within the circle shown in the middle column of Fig. 10.

On the right side of Fig. 10, we show the color-magnitude diagram for stars assumed to be cluster members. The available archive images are again not sufficiently deep to derive reliable proper motions below the TO. The proper motions presented in this paper are not sufficiently accurate to study the internal motion of $\omega$ Cen. The main purpose of the proper motion presented in this work is to provide a reliable membership probability for spectroscopic follow-up projects, star counts, and the study of the radial distribution of the different branches (Bellini et al., in preparation).

\subsection{Astrometric calibration}

To translate the pixel coordinates into the equatorial coordinate system, we adopted the UCAC2 catalog (Zacharias et al. 2004) as a reference frame. Due to the severe crowding in images of the inner parts of $\omega$ Cen, this catalog was however inadequate for calibration purposes close to the center of $\omega$ Cen (the central $10^{\prime} \times 10^{\prime}$ area corresponds almost entirely to a void in UCAC2). Another possible reference frame, especially for the cluster center, is the vL00 catalog. However, the precision of published coordinates is lower than $\sim 20$ mas and no analysis was provided by vL00 for the presence of potential systematic errors in the positions. Examination of vL00 proper motions by Platais et al. (2003) indicated that a priori these systematic errors could not be discounted. These deficiencies in the vL00 positional catalog were eliminated by re-reducing the original Cartesian coordinates (of formal precision equal to 2 mas), kindly provided by van Leeuwen. 

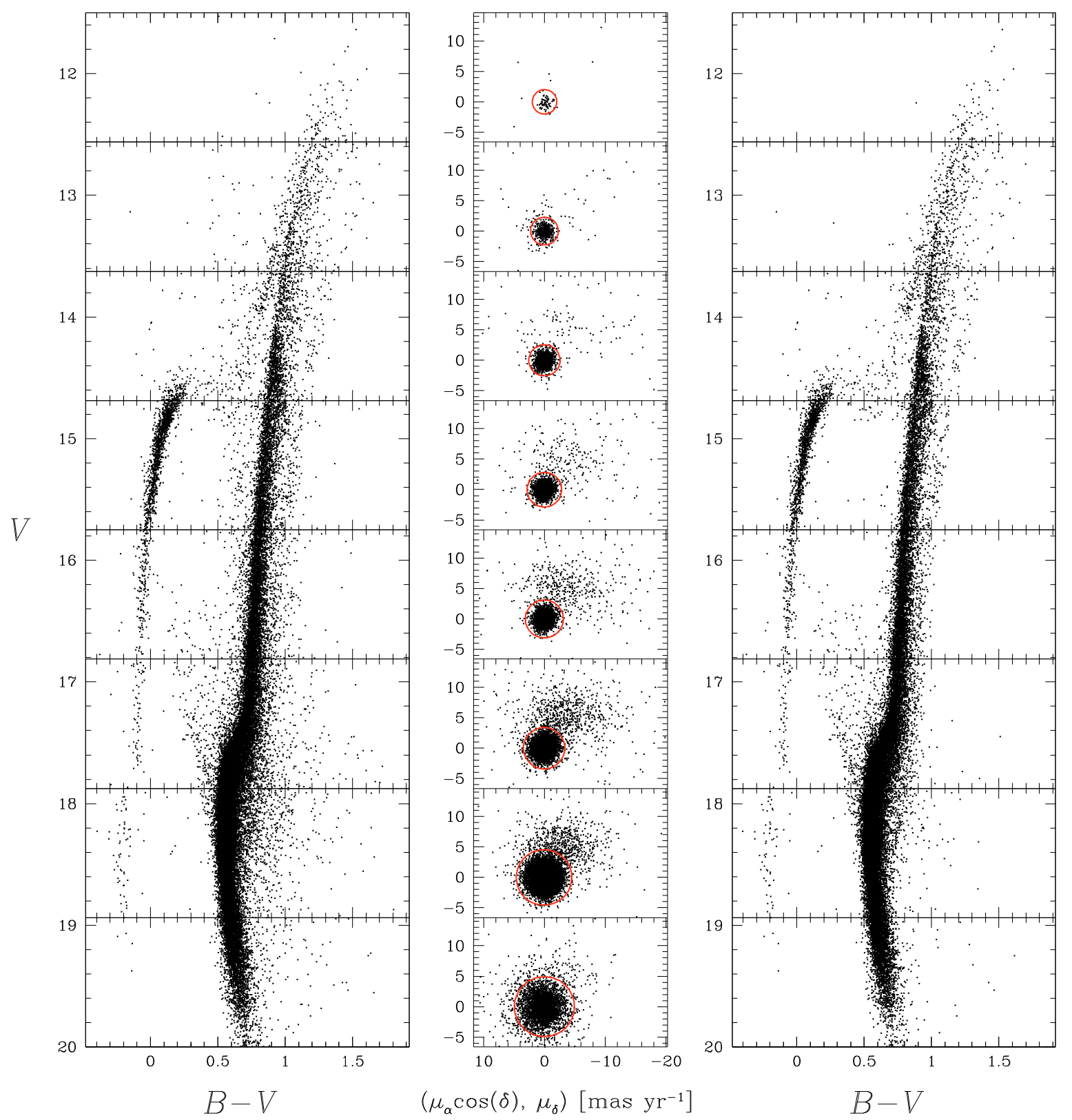

Fig. 10. Left: color-magnitude diagram, splitted into eight magnitude bins, for all the stars having a proper-motion rms increasing from 1.8 mas $\mathrm{yr}^{-1}$ for the brightest bin to $5 \mathrm{mas} \mathrm{yr}^{-1}$ for the faintest one, and photometric rms from $0.02 \mathrm{mag}$ to $0.05 \mathrm{mag}$, generous enough to include main sequence stars down to $V=20$. Middle: proper motions for the stars in the corresponding magnitude intervals. A circle in each diagram shows the adopted membership criterion. Right: color-magnitude diagram for assumed cluster members.

First, we selected only Class $0-1$ stars from vL00 (i.e. their images were isolated or only slightly disturbed by an adjacent image). Second, a trial equatorial solution was obtained for vL00 stars using the UCAC2 catalog as a reference frame. Third, the new set of vL00 coordinates was tested against the UCAC2 positions as a function of coordinates, magnitude, and color of stars. There are $\sim 3000$ stars in common between these two sets of coordinates. Assuming that the UCAC2 positions are free of magnitude-color-related systematic errors, we found that the original vL00 Cartesian coordinates were biased by up to $16 \mathrm{mas}^{\mathrm{mag}}{ }^{-1}$. There is also a detectable quadratic colordependent bias along the declination. Both magnitude- and color-related biases were removed from the vL00 Cartesian coordinates before the final equatorial solution was obtained.
The new reference catalog, covering a region of $1.5 \times 1.5$ and magnitudes to $V \sim 16.5$, contains 10291 stars and consists of approximately equal parts of the UCAC2 (trimmed down to stars with positional accuracies of higher quality than 75 mas) and the updated vL00 coordinates on the system of ICRS and epoch J2000.0.

This new reference catalog was used to obtain the equatorial coordinates of our $\omega$ Cen stars. The WFI pixel coordinates of these stars were translated into global Cartesian system coordinates and corrected for geometric distortions. A simple low-term-dominated plate model was sufficient to calculate equatorial coordinates. The standard error of this solution, employing $\sim 5500$ reference stars, was $45-50$ mas in each coordinate. These errors were higher than those listed in Yadav et al. (2008, Paper II), which is based on similar WFI@2.2m data for 

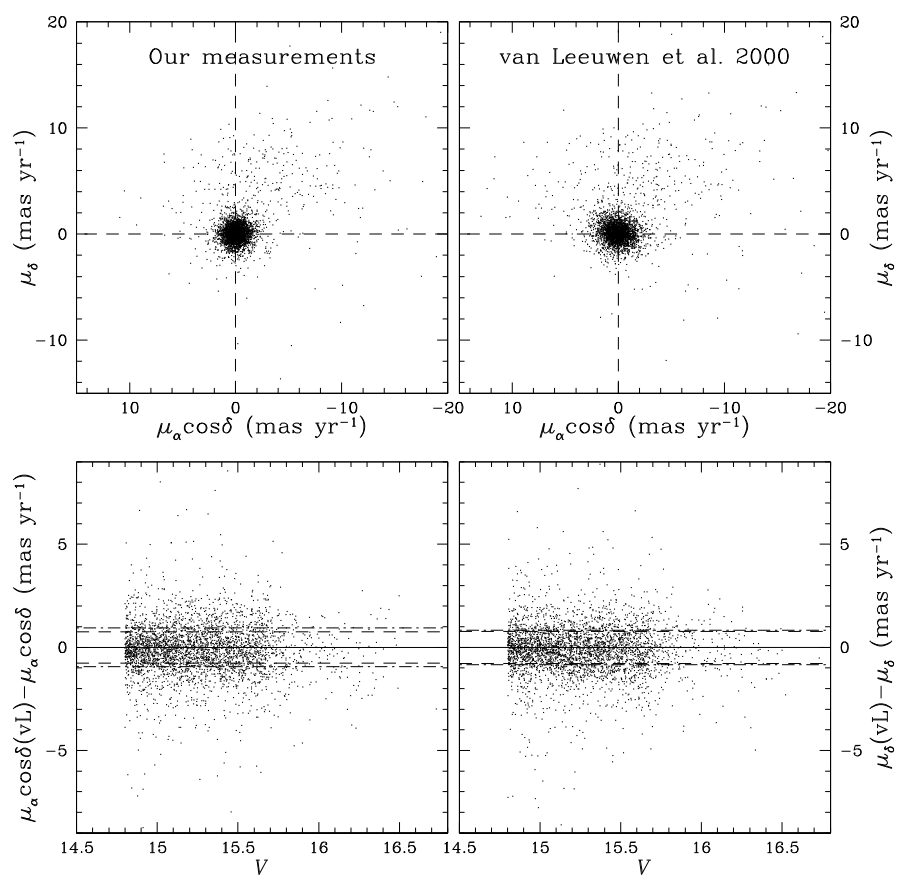

Fig. 11. Top panels: vector-point diagrams of common stars in our catalog left and those of vL00 (right), with respect to the mean cluster motion. Bottom panels: right ascension left and declination right propermotion residuals as a function of the $V$ magnitudes. Horizontal dashed and point-dashed lines show the $3 \sigma$-clipped median of the propermotion dispersion for the selected cluster members, for our measurements and for vL00, respectively.

the open cluster M67. We understand that image crowding remains a dominant source of increased scatter in our solution for $\omega$ Cen. Although we removed all stars with obviously poor astrometry, even a close but not overlapping image might slightly distort the position of a star, especially in photographic plates. The J2000 positions of all stars for the epoch 2003.29 are given in Table 6.

The proper motions in this work have not been translated into the absolute values, because there are too few background galaxies suitable for defining an absolute reference frame.

\subsection{Comparison with other $\omega$ Cen proper-motion catalogs}

We compare our results with the proper-motion catalog by van Leeuwen (2000). First, we considered the common, unsaturated stars in our catalog $(V>14.6)$ to the vL00 faint limit $(V \sim 16.5)$. The selected samples contained $\sim 3400$ stars. Since vL00 proper motions are given in an absolute reference system, we subtracted from the individual vL00 proper motions the absolute mean motion of the cluster provided by the same authors $\left[\left(\mu_{\alpha} \cos \delta, \mu_{\delta}\right)=(-3.97,-4.38)\right.$ mas yr$\left.^{-1}\right]$.

In the top panels of Fig. 11, we show on the left the vectorpoint diagram from our measurements, while on the right we show the vL00 values. In both diagrams, a concentration of stars

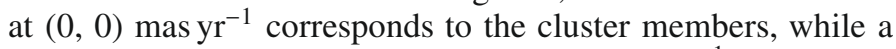
diffuse distribution of stars around $(-3,5)$ mas $^{-1} \mathrm{r}^{-1}$, consists of the field objects in the foreground and possibly the background of $\omega$ Cen.

The size of the proper-motion dispersions of the cluster members reflect both internal motions in the cluster and random errors. However, the internal motions are expected to be negligible. Assuming a distance of $5.5 \mathrm{kpc}$, and the Meylan et al. (1995) measurement of the dispersion in the transverse velocity of $\sim 10 \mathrm{~km} \mathrm{~s}^{-1}$ (in the outskirts of the cluster that we are probing) the expected dispersion in the proper motions would be $\sim 0.4 \mathrm{mas} \mathrm{yr}^{-1}$. Our estimated errors for the selected sample was $0.74 \mathrm{mas} \mathrm{yr}^{-1}$ for $\mu_{\alpha} \cos \delta$, and $0.77 \mathrm{mas} \mathrm{yr}^{-1}$ for $\mu_{\delta}$. Therefore, the internal proper motions should not affect more than $10-15 \%$ of the observed dispersions.

To estimate the observed proper-motion dispersion in the two samples (this paper and vL00), we adopted the 68.27th percentile of the distribution $(\sigma)$ about the median (estimated iteratively with a $3 \sigma$-clipping), and for each coordinate independently. Due to the significance differences between cluster and field object motion, this procedure allowed us to isolate a sub sample of members.

Our results were:

this work : $\left\{\begin{array}{l}\sigma\left(\mu_{\alpha} \cos \delta\right)=0.76 \mathrm{mas} \mathrm{yr}^{-1} \\ \sigma\left(\mu_{\delta}\right)=0.78 \mathrm{mas} \mathrm{yr}^{-1}\end{array}\right.$

vL00 : $\left\{\begin{array}{l}\sigma\left(\mu_{\alpha} \cos \delta\right)=0.94{\text { mas } \mathrm{yr}^{-1}}^{-1} \\ \sigma\left(\mu_{\delta}\right)=0.83 \mathrm{mas} \mathrm{yr}^{-1}\end{array}\right.$

For the selected sample, it is clear that our distribution is tighter, rounder, and in good agreement with our estimate of the errors. Even if our proper motions originate in images representing half the total number of plates used by vLO0, we note that we study more than 3 mag fainter in $V$, and use a time baseline that equals only $\sim 1 / 12$ of that used by vL00.

The above performed test could be a bit unfair versus the vL00 catalog, because we used our non-saturated stars only, which are the faintest in the vL00 catalog. vL00 demonstrated emphatically that not all stars are suitable for astrometric measurements. We therefore performed a second test in which we chose the stars in vL00 with the most reliable measurements (belonging to class 0 and 1 only) that were brighter than $V=16$, and had a good probability of being a cluster member $\left(P_{\mu}(\mathrm{vLO0})>\right.$ $75 \%$ ); we compared the measurements for these stars with those in catalog.

The results were as follows:

this work : $\left\{\begin{array}{l}\sigma\left(\mu_{\alpha} \cos \delta\right)=0.68 \mathrm{mas} \mathrm{yr}^{-1} \\ \sigma\left(\mu_{\delta}\right)=0.69 \mathrm{mas} \mathrm{yr}^{-1}\end{array}\right.$

vL00 : $\left\{\begin{array}{l}\sigma\left(\mu_{\alpha} \cos \delta\right)=0.71 \text { mas yr}^{-1} \\ \sigma\left(\mu_{\delta}\right)=0.62{\text { mas } \mathrm{yr}^{-1}}\end{array}\right.$

These results illustrate the slightly higher precision of the vL00 catalog, but the dispersions $\mathrm{n}$ the measurements are, in any cases, comparable. The dispersion obtained with our catalog, which now also includes saturated star measurements, is more reliable than found with the first test. The explanation of this apparent paradox is that, by selecting the best proper-motion measured stars in the vL00 catalog (class 0 and 1, among the most isolated stars), we also selected the most isolated stars in our catalog, making the PSF-wings fitting more effective. Once again, it appears clear the huge potential that wide field CCD imager will have in astrometry in the future.

\section{Membership probability}

In the vector-point diagrams of Figs. 8 and 10, two distinct groups of stars are clearly detectable: the bulk of stars belong to $\omega$ Cen, with no mean motion $\left(\mu_{\alpha} \cos (\delta)=\mu_{\delta}=0.0\right.$ mas yr$\left.^{-1}\right)$, and there is a secondary broad group, which corresponds to field stars. 

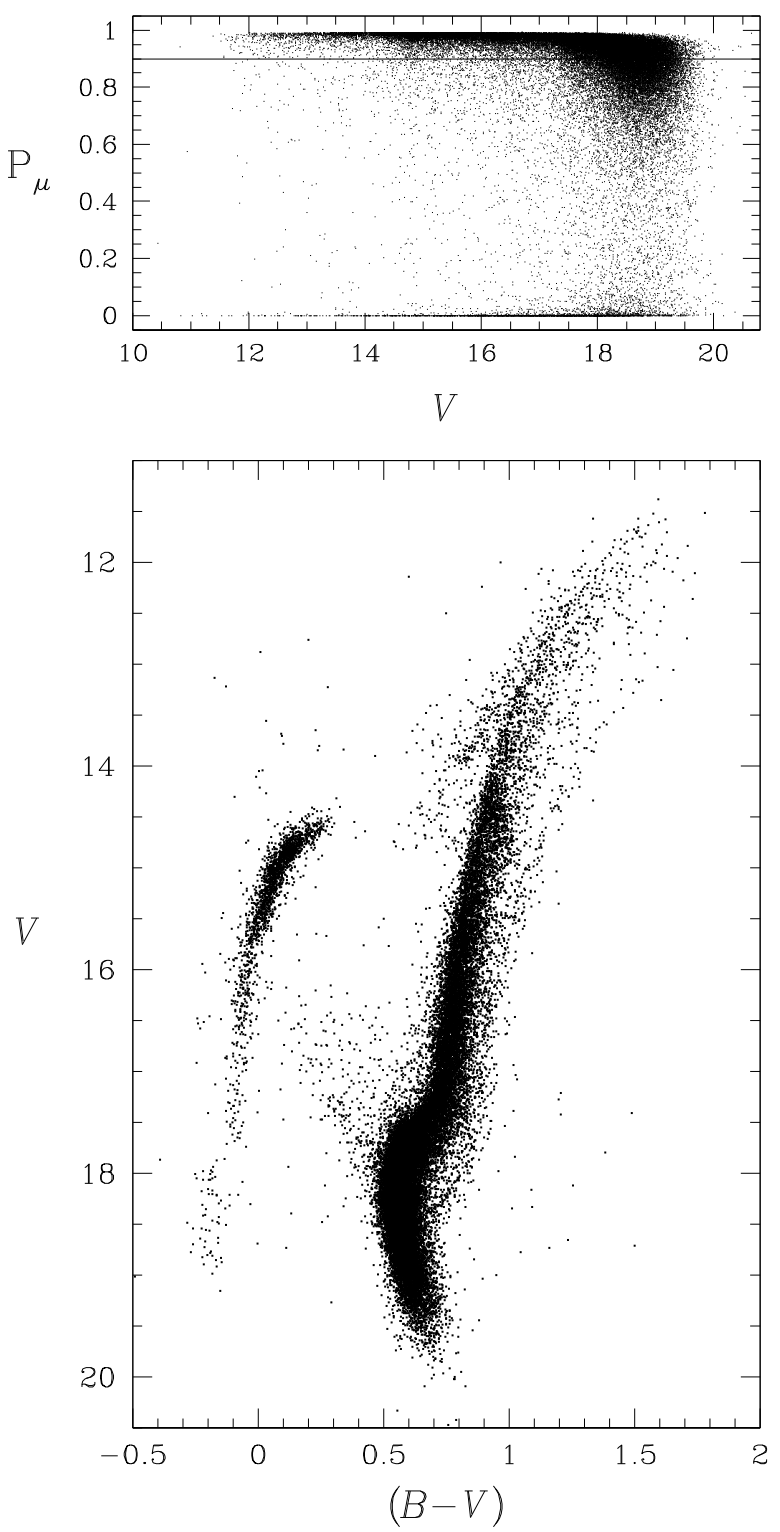

Fig. 12. Membership probability $P_{\mu}$ versus $V$ magnitude for stars with

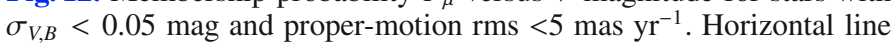
show the $P_{\mu}=90 \%$ level. The resulting CMD for stars with $P_{\mu}>90 \%$ is shown on the bottom panel.

Vasilevskis et al. (1958) were the first to formulate the proper-motion membership probability. This method was later developed by many authors (Sanders 1971; Zhao \& He 1990; Tian et al. 1998; Balaguer-Núnez et al. 1998, and references therein) for several open and globular clusters. To derive our membership probability, we followed a method based on proper motions described by Balaguer-Núnez et al. (1998).

First of all, we constructed frequency functions for both cluster and field stars, $\Phi_{\mathrm{c}}^{v}$ and $\Phi_{\mathrm{f}}^{v}$ respectively, derived from the cluster and field star distribution in the VPD. For the $i$ th star, these functions were as follows:

$$
\Phi_{\mathrm{c}}^{v}=\frac{\exp \left\{-\frac{1}{2}\left[\frac{\left(\mu_{x_{i}}-\mu_{x_{\mathrm{c}}}\right)^{2}}{\sigma_{x_{\mathrm{c}}}^{2}+\epsilon_{x_{i}}^{2}}+\frac{\left(\mu_{y_{i}}-\mu_{y_{\mathrm{c}}}\right)^{2}}{\sigma_{y_{\mathrm{c}}}^{2}+\epsilon_{y_{i}}^{2}}\right]\right\}}{2 \pi\left(\sigma_{x_{\mathrm{c}}}^{2}+\epsilon_{x_{i}}^{2}\right)^{1 / 2}\left(\sigma_{y_{\mathrm{c}}}^{2}+\epsilon_{y_{i}}^{2}\right)^{1 / 2}}
$$

and

$\Phi_{\mathrm{f}}^{v}=\frac{\exp \left\{-\frac{1}{2\left(1-\gamma^{2}\right)} \cdot\left[\frac{\left(\mu_{x_{i}}-\mu_{x_{\mathrm{f}}}\right)^{2}}{\sigma_{x_{\mathrm{f}}}^{2}+\epsilon_{x_{i}}^{2}}-\frac{2 \gamma\left(\mu_{x_{i}}-\mu_{x_{\mathrm{f}}}\right)\left(\mu_{y_{i}}-\mu_{y_{\mathrm{f}}}\right)}{\left(\sigma_{x_{\mathrm{f}}}^{2}+\epsilon_{x_{i}}^{2}\right)^{1 / 2}\left(\sigma_{y_{\mathrm{f}}}^{2}+\epsilon_{y_{i}}^{2}\right)^{1 / 2}}+\frac{\left(\mu_{y_{i}}-\mu_{y_{\mathrm{f}}}\right)^{2}}{\sigma_{y_{\mathrm{f}}}^{2}+\epsilon_{y_{i}}^{2}}\right]\right\}}{2 \pi\left(1-\gamma^{2}\right)^{1 / 2}\left(\sigma_{x_{\mathrm{f}}}^{2}+\epsilon_{x_{i}}^{2}\right)^{1 / 2}\left(\sigma_{y_{\mathrm{f}}}^{2}+\epsilon_{y_{i}}^{2}\right)^{1 / 2}}$

where $\left(\mu_{x_{i}}, \mu_{y_{i}}\right)$ are the $i$ th star proper motions along the $X$ and $Y$ axes, $\left(\epsilon_{x_{i}}, \epsilon_{y_{i}}\right)$ represent the respective displacement rms, $\left(\mu_{x_{\mathrm{f}}}, \mu_{y_{\mathrm{f}}}\right)$ and $\left(\mu_{x_{\mathrm{c}}}, \mu_{y_{\mathrm{c}}}\right)$ are the central points of the field and cluster star proper motion, $\left(\sigma_{x_{\mathrm{f}}}, \sigma_{y_{\mathrm{f}}}\right)$ and $\left(\sigma_{x_{\mathrm{c}}}, \sigma_{y_{\mathrm{c}}}\right)$ are the field and cluster star proper-motion intrinsic dispersion, and $\gamma$ is the correlation coefficient, calculated to be

$$
\gamma=\frac{\left(\mu_{x_{i}}-\mu_{x_{\mathrm{f}}}\right)\left(\mu_{y_{i}}-\mu_{y_{\mathrm{f}}}\right)}{\sigma_{x_{\mathrm{f}}} \sigma_{y_{\mathrm{f}}}} .
$$

The spatial distribution was ignored due to the relatively small size of our field $\left(\sim 33^{\prime} \times 33^{\prime}\right)$ with respect to the $\omega$ Cen radial extent of $r_{t} \simeq 57^{\prime}$ (Harris et al. 1996). For our calculations, we considered only stars with an rms in proper motion

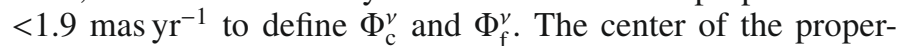
motion distribution in the VPD for cluster stars was found to be at $x_{\mathrm{c}}=0.00 \mathrm{mas} \mathrm{yr}^{-1}$, and $y_{\mathrm{c}}=0.00 \mathrm{mas} \mathrm{yr}^{-1}$, with $\mathrm{rms}$ values of $\sigma_{x_{\mathrm{c}}}=0.83 \mathrm{mas} \mathrm{yr}^{-1}$, and $\sigma_{y_{\mathrm{c}}}=0.83 \mathrm{mas} \mathrm{yr}^{-1}$. For field stars, we have: $x_{\mathrm{f}}=-3.57 \mathrm{mas} \mathrm{yr}^{-1}, y_{\mathrm{f}}=5.12 \mathrm{mas} \mathrm{yr}^{-1}$, $\sigma_{x_{\mathrm{f}}}=5.06 \mathrm{mas} \mathrm{yr}^{-1}$, and $\sigma_{y_{\mathrm{f}}}=2.86 \mathrm{mas} \mathrm{yr}^{-1}$, respectively.

The distribution function for all the stars can be computed as follows:

$\Phi=\left(n_{\mathrm{c}} \cdot \Phi_{\mathrm{c}}^{v}\right)+\left(n_{\mathrm{f}} \cdot \Phi_{\mathrm{f}}^{v}\right)=\Phi_{\mathrm{c}}+\Phi_{\mathrm{f}}$

where $n_{\mathrm{c}}$ and $n_{\mathrm{f}}$ are the normalized number of stars for cluster and field $\left(n_{\mathrm{c}}+n_{\mathrm{f}}=1\right)$. Therefore, for the $i$ th star the resulting membership probability is

$P_{\mu}(i)=\frac{\Phi_{\mathrm{c}}(i)}{\Phi(i)}$

In Fig. 12, we show in the upper panel the $P_{\mu}$ distribution versus $V$ magnitude. To include also faint stars and reach $V=20$,

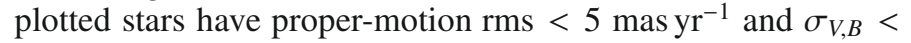
0.05 mag. The horizontal line marks the $90 \%$ probability level. The lower panel contains the CMD for stars with $P_{\mu}>90 \%$.

When calculating formal membership probabilities for $\omega$ Cen, we have a paradoxical situation in which the main concern is to assign a reasonable probability to field stars. This is because in our sample the number of cluster stars is significantly higher than the small number of field stars. In addition, proper-motion errors have a strong dependence on magnitude (Figs. 7, 10), which is not accounted for in our membership probability $P_{\mu}$ calculation. We therefore, also used the so-called local sample method (e.g., Paper II) for membership probability calculation. In this method, for each target star a sub-sample of stars was selected to reflect closely the properties of a target. This assures a smooth transition in the calculated $P_{\mu}$ as a function of the chosen parameter. For $\omega$ Cen, the obvious choice of parameter was the mean error $\sigma_{\mu}$ of the proper motions. Given the wide range of $\sigma_{\mu}$, we chose to consider only stars with

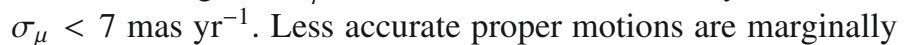
useful for membership studies. We note that $\sigma_{\mu}$ is calculated in the same way as in Sect. 4 and Fig. 9.

For each target star, we then selected a star a subsample of 3000 stars almost with identical proper-motion errors to that of a target star. The trial calculations indicated that we cannot model the distribution of field stars with a Gaussian because the number of potential field stars in the vector-point diagram is extremely 


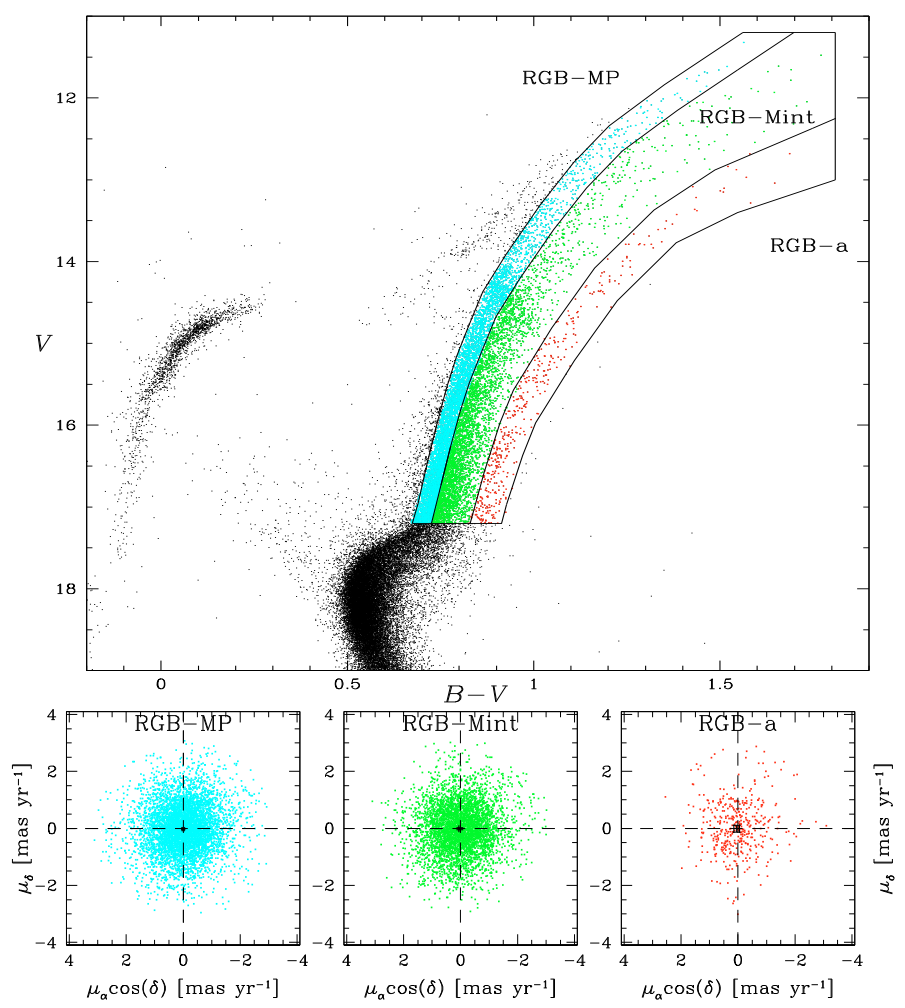

Fig. 13. Top panel: $V$ vs. $B-V$ CMD of proper-motion-selected member stars on which we defined three RGB subsamples: RGB-MP (cyan), RGB-Mint (green), and RGB-a (red). See text for the definition of the subpopulations. Bottom panels: proper motions of RGB-MP left, RGBMint middle, and RGB-a (right) subpopulations.

low in the vicinity of the cluster-star centroid (Fig. 11). A reasonable alternative to a Gaussian is a flat distribution of field stars.

In essence, the membership probability $P_{\mu}$ is driven by the distribution of cluster stars. If the modulus of the proper-motion vector of a star exceeds $2.5-3 \sigma_{\mu}$, the corresponding $P_{\mu}$ is less than $1 \%$. We provide these alternative estimates of $P_{\mu}$ (called $P_{\mu}(2)$, to distinguish this from the first mentioned method $\left.P_{\mu}(1)\right)$ for 120259 stars. For the majority of stars, both values of $P_{\mu}$ are similar. However, there are a number of cases in which the $P_{\mu}$ estimates for the two methods differ radically. In the case of high proper-motion errors for cluster stars, the local sample method clearly provides a more realistic membership probability. A closer inspection of these cases indicates indirectly a potential problem in calculating the proper motion. If the error of proper motion along one axis is several times larger than the error along the other axis, or if this error is too high for a particular magnitude, the chances are that our proper-motion value is corrupted and, hence, its formal membership probability is meaningless.

Unless specified otherwise, we mean $P_{\mu}(1)$ determination when referring to $P_{\mu}$.

\section{Applications}

Our $\omega$ Cen proper-motion catalog can be used for different purposes. The first application was the selection of the most probable $\omega$ Cen members for spectroscopic follow-up studies. In Villanova et al. (2007), we used the proper-motion catalog of the present paper to pre-select sub-giant branch stars. This helped us to avoid the Galactic field stars close to the TO level. All resultant radial velocities were close to the $\omega$ Cen mean value, which confirmed their membership. On the other hand, the high photometric quality and the availability of several filters covering a wide area around the cluster, imply that this catalog is an excellent photometric reference frame on which to register photometry from different telescopes and cameras (Bellini et al., in preparation).

Besides these obvious applications, our $\omega$ Cen propermotion catalog also provides an observational constraint of the origin of the composite stellar populations in $\omega$ Cen. Our catalog provides the necessary wide-field coverage and photometric accuracy to investigate the radial distribution of the different $\omega$ Cen sub-population from the center of the cluster to $\sim 22^{\prime}$ (in the corners). We will report on this analysis in a subsequent paper (Bellini et al., in preparation).

\subsection{The proper motion of the RGB sub-populations}

Ferraro et al. (2002) cross-correlated the WFI photometric catalog of Pancino et al. (2000) with the vL00 photographic propermotion catalog. Their goal was to investigate the nature of the anomalous metal-rich RGB of $\omega$ Cen, the so-called RGB-a. In particular, they investigated the presence of proper-motion mean differences between the $\omega$ Cen bulk population (metalpoor RGB, so-called RGB-MP) and the minor, but yet important, metal-rich RGB-a population (Pancino et al. 2000). Their Fig. 2 showed significant variation in the relative proper motion of RGB-a stars with respect to $\omega$ Cen RGB-MP motion. In particular, they found that the RGB-a stars had a mean proper motion of $\left|\delta \mu_{\text {tot }}\right|=0.8$ mas yr $^{-1}$ that is offset from that of the RGB-MP population. Therefore, they concluded that the RGB-a subpopulation must have had an independent origin with respect to the RGB-MP one.

Unsurprisingly, the Ferraro et al. (2002) study triggered new interest in the $\omega$ Cen proper motion, and Platais et al. (2003) presented a detailed reanalysis of the vL00 catalog. Platais et al. (2003) concluded that the reported proper-motion offset between the $\omega$ Cen sub-populations could be attributed to instrumental bias. However, Hughes et al. (2004) commented that there was no residual color term in the omega Cen proper motions, and that these authors misinterpreted the observed offsets. Specifically, Hughes et al. (2004) asserted that the summary effect of color terms (before the corrections) amounted to no more than $1 \mathrm{mas} \mathrm{yr}^{-1} \mathrm{mag}^{-1}$ in $B-V$, while the offset in the proper motions for the anomalous omega Cen stars reached 2 mas yr$^{-1}$ and did not have the same direction as the color term. Regardless of the reason for the reported offset in the vL00 catalog, the presence of this offset was not confirmed by the new HST-based preliminary proper motions (Anderson 2003).

Two spectroscopic studies completed by Pancino et al. (2007) and Johnson et al. (2008) indicated that there was no evidence for any of the RGB stellar populations to have an offset in the mean radial velocity, or a different radial velocity dispersion. This result applied also to the RGB-a sub-population.

Our astrometric catalog provided an independent data set with which we could test the Ferraro et al. (2002) conclusions. We repeated the same analysis performed in Ferraro et al. (2002). First, we divided the $\omega$ Cen RGB population into three subpopulations (see top panel of Fig. 13): RGB-MP (cyan), RGB-a (red), and RGB-Mint (RGB stars between RGB-MP and RGB-a, green). All plotted stars had high membership probability $\left(P_{\mu}>90 \%\right)$, and photometric errors ranging from $0.02 \mathrm{mag}$ for bright stars to 0.05 mag for faintest ones, in both filters. 


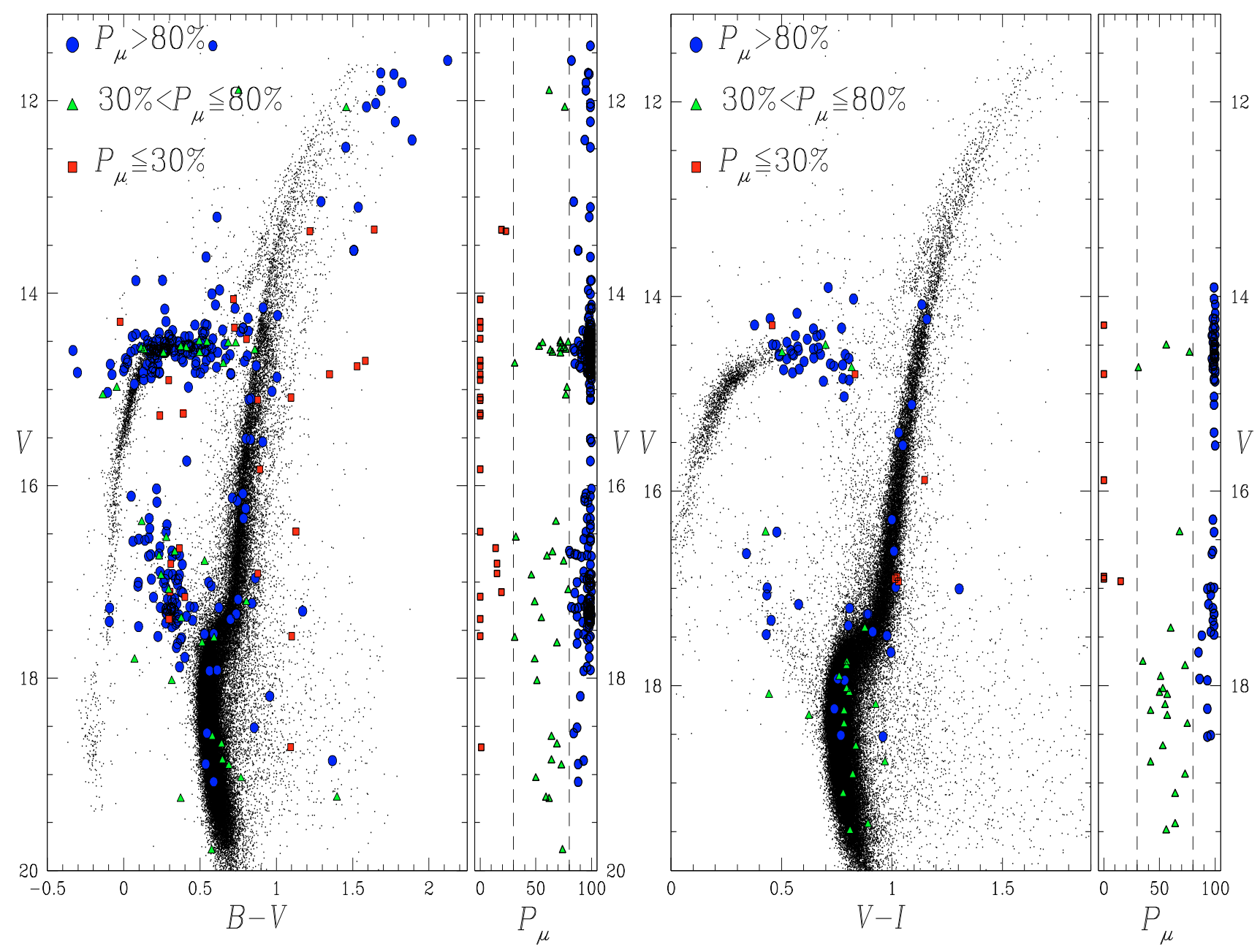

Fig. 14. Left: $\omega$ Cen CMD with the cross-checked variable stars from the Kaluzny et al. (2004) catalog; red squares mark stars with a membership probability $P_{\mu}<30$; green triangles are stars with $30 \leq P_{\mu}<80$, while blue circles are those with a probability to be cluster members $P_{\mu} \geq 80$. The membership probability $P_{\mu}$ versus the $V$ magnitude is also shown. Right: comparison with Weldrake et al. (2007) catalog.

The RGB samples include all stars with $V \leq 17.2$. MP and Mint RGB stars merge with each other at fainter magnitudes. Sollima et al. (2005) and Villanova et al. (2007) clearly showed that the SGB region of $\omega$ Cen includes five subpopulations (see again Fig. 15). Three of these merge into each other and form the RGB-MP and RGB-Mint samples. We emphasize that our population sub-division extends to fainter magnitudes than in Ferraro et al. (2002), and therefore provides a higher RGB sampling and more robust statistics. Our samples contain 5182 RGBMP stars, 3127 RGB-Mint stars, and 313 RGB-a stars.

A closer look at the three RGB sub-population proper motions is presented in the bottom panels of Fig. 13: RGB-MP on the left, RGB-Mint in the middle, and RGB-a on the right. The red crosses report the median value (estimated iteratively) of the proper motion of the three sub-samples. For the RGB-MP sample, we have:

$\left\{\begin{array}{l}\left\langle\mu_{\alpha} \cos \delta\right\rangle=(0.00 \pm 0.01) \text { mas }^{-1} r^{-1} \\ \left\langle\mu_{\delta}\right\rangle=(-0.02 \pm 0.01){\text { mas } \mathrm{yr}^{-1}}\end{array}\right.$

for RGB-Mint stars:

$\left\{\begin{array}{l}\left\langle\mu_{\alpha} \cos \delta\right\rangle=(0.03 \pm 0.01) \mathrm{mas} \mathrm{yr}^{-1} \\ \left\langle\mu_{\delta}\right\rangle=(-0.01 \pm 0.01) \text { mas } \mathrm{yr}^{-1},\end{array}\right.$ and for the RGB-a sample:

$\left\{\begin{array}{l}\left\langle\mu_{\alpha} \cos \delta\right\rangle=(0.05 \pm 0.03) \mathrm{mas}^{-1} \\ \left\langle\mu_{\delta}\right\rangle=(-0.01 \pm 0.04) \mathrm{mas} \mathrm{yr}^{-1}\end{array}\right.$

We found no evidence for the presence of differences among the relative proper motions of the three RGB sub-populations at the

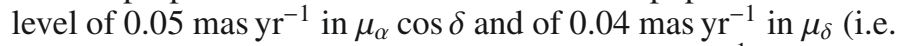
relative tangential velocities of $\lesssim 1.3$, and $\lesssim 1.1 \mathrm{~km} \mathrm{~s}^{-1}$, assuming a distance of $5.5 \mathrm{kpc}$ for $\omega \mathrm{Cen}$ ). All three RGB sub-samples exhibit the same mean proper motion, within the errors.

We therefore, agree with the results of both Platais et al. (2003) and Johnson et al. (2008) for RGB-a, and show that the RGB-Mint proper motion is also consistent with the other $\omega$ Cen sub-populations.

A final word on this issue requires an internal stellar propermotion analysis, but suitable catalogs are not yet available.

\subsection{Membership probability of published $\omega$ Cen variable stars}

The study of variable stars in $\omega$ Cen can certainly benefit from our proper-motion catalog and cluster membership derivation. 
Table 3. Membership probability for the Kaluzny et al. (2004) variable star catalog. ID $_{\mathrm{K}}$ are the Kaluzny identification labels, while ID ${ }_{\text {tw }}$ refer to this work.

\begin{tabular}{|c|c|c|c|c|c|c|c|c|c|c|c|c|c|c|c|c|c|}
\hline$\overline{\mathrm{ID}_{\mathrm{K}}}$ & 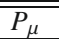 & $\overline{\mathrm{ID}_{\mathrm{tw}}}$ & $\overline{\mathrm{ID}_{\mathrm{K}}}$ & $\overline{\bar{P}}$ & $\overline{\mathrm{ID}_{\mathrm{tw}}}$ & $\overline{\mathrm{ID}_{\mathrm{K}}}$ & $\overline{P_{\mu}}$ & $\overline{\mathrm{ID}_{\mathrm{tw}}}$ & $\overline{\mathrm{ID}_{\mathrm{K}}}$ & $\overline{\bar{P}}$ & $\overline{\mathrm{ID}_{\mathrm{tw}}}$ & $\overline{\mathrm{ID}_{\mathrm{K}}}$ & $\overline{P_{\mu}}$ & $\overline{\mathrm{ID}_{\mathrm{tw}}}$ & $\overline{\mathrm{ID}_{\mathrm{K}}}$ & $\overline{\bar{P}}$ & $\overline{\mathrm{ID}_{\mathrm{tw}}}$ \\
\hline V1 & 99 & 290466 & V66 & $\mu 9$ & 311494 & V132 & $\mu$ & 184072 & V214 & 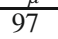 & 42989 & NV296 & $\begin{array}{l}\mu 9 \\
99\end{array}$ & 356415 & NV354 & 97 & 263543 \\
\hline V2 & 74 & 271987 & V67 & 99 & 352882 & V135 & 97 & 181199 & V216 & 99 & 155556 & NV297 & 32 & 250290 & NV355 & 93 & 196052 \\
\hline V3 & 99 & 248511 & V68 & 100 & 345639 & V136 & 99 & 213549 & V217 & 98 & 218342 & NV298 & 100 & 87382 & NV356 & 49 & 206069 \\
\hline V4 & 97 & 279540 & V70 & 98 & 93459 & V137 & 99 & 225149 & V218 & 96 & 358729 & NV299 & 98 & 310461 & NV357 & 92 & 158687 \\
\hline V5 & 100 & 298983 & V71 & 99 & 209884 & V139 & 90 & 215311 & V219 & 88 & 347441 & NV300 & 98 & 284928 & NV358 & 99 & 129801 \\
\hline V8 & 99 & 200194 & V74 & 100 & 360819 & V141 & 100 & 177819 & V220 & 100 & 321724 & NV301 & 96 & 170554 & NV359 & 46 & 222238 \\
\hline V9 & 100 & 238167 & V75 & 99 & 352935 & V142 & 65 & 193092 & V221 & 96 & 320315 & NV302 & 19 & 252220 & NV360 & 100 & 118132 \\
\hline V10 & 98 & 273997 & V76 & 100 & 340408 & V143 & 90 & 217472 & V223 & 0 & 355759 & NV303 & 98 & 145690 & NV361 & 64 & 234998 \\
\hline V11 & 99 & 302079 & V77 & 99 & 316247 & V144 & 95 & 201059 & V224 & 99 & 349215 & NV304 & 98 & 341374 & NV363 & 93 & 321474 \\
\hline V12 & 99 & 283465 & V78 & 0 & 243506 & V145 & 91 & 143665 & V225 & 96 & 274338 & NV305 & 0 & 343091 & NV364 & 93 & 243121 \\
\hline V13 & 99 & 259326 & V81 & 99 & 270509 & V146 & 72 & 177889 & V226 & 99 & 167657 & NV306 & 96 & 198996 & NV365 & 0 & 71679 \\
\hline V14 & 97 & 26311 & V82 & 99 & 236315 & V147 & 97 & 143269 & V227 & 98 & 287148 & NV307 & 82 & 211960 & NV366 & 97 & 132355 \\
\hline V15 & 100 & 273754 & V83 & 99 & 323500 & V148 & 95 & 209005 & V228 & 96 & 92375 & NV308 & 98 & 327308 & NV367 & 0 & 350053 \\
\hline V16 & 100 & 40312 & V85 & 98 & 292312 & V150 & 100 & 58317 & V229 & 97 & 285982 & NV309 & 85 & 193994 & NV368 & 0 & 349013 \\
\hline V17 & 99 & 261135 & V86 & 99 & 242948 & V152 & 82 & 177559 & V231 & 99 & 59688 & NV310 & 15 & 127228 & NV369 & 94 & 124330 \\
\hline V18 & 98 & 267824 & V87 & 97 & 255154 & V153 & 100 & 238719 & V233 & 99 & 336053 & NV311 & 91 & 170132 & NV370 & 0 & 358442 \\
\hline V19 & 100 & 205260 & V88 & 94 & 261450 & V154 & 96 & 155744 & V234 & 0 & 157490 & NV312 & 95 & 183516 & NV372 & 93 & 290428 \\
\hline V20 & 99 & 204861 & V90 & 89 & 239287 & V155 & 98 & 272492 & V235 & 87 & 309505 & NV313 & 98 & 134090 & NV373 & 59 & 300775 \\
\hline $\mathrm{V} 21$ & 99 & 246994 & V91 & 92 & 241579 & V156 & 99 & 128871 & V236 & 85 & 282601 & NV314 & 85 & 163946 & 74 & 99 & 312254 \\
\hline V22 & 99 & 86072 & V92 & 99 & 327526 & V157 & 98 & 221081 & V237 & 99 & 286453 & V315 & 60 & 267539 & 1375 & 0 & 349760 \\
\hline V23 & 100 & 273446 & V94 & 98 & 305927 & V158 & 31 & 153087 & V238 & 99 & 179489 & NV316 & 99 & 142842 & NV376 & 0 & 189233 \\
\hline V24 & 99 & 84169 & V95 & 94 & 188847 & V161 & 76 & 149737 & V239 & 99 & 179912 & NV317 & 100 & 361100 & NV377 & 0 & 324933 \\
\hline V25 & 99 & 199162 & V96 & 63 & 225607 & V162 & 97 & 108440 & V240 & 99 & 239985 & NV318 & 98 & 246801 & 78 & 31 & 341736 \\
\hline V26 & 98 & 226616 & V97 & 99 & 256623 & V163 & 100 & 337663 & V241 & 1 & 289203 & NV319 & 94 & 145544 & NV379 & 99 & 328605 \\
\hline V27 & 96 & 201410 & V98 & 99 & 227653 & V164 & 99 & 334394 & V242 & 98 & 171250 & NV320 & 99 & 80363 & NV380 & 96 & 148727 \\
\hline V29 & 62 & 191401 & V99 & 99 & 210796 & V165 & 85 & 227981 & V249 & 99 & 236550 & NV321 & 87 & 209026 & NV381 & 0 & 311380 \\
\hline V30 & 99 & 168094 & V100 & 77 & 215927 & V166 & 72 & 241584 & V250 & 92 & 62410 & NV322 & 93 & 214126 & NV382 & 96 & 343538 \\
\hline V32 & 100 & 322448 & V101 & 99 & 169863 & V167 & 23 & 87332 & V251 & 97 & 231812 & NV323 & 65 & 265232 & NV383 & 99 & 336713 \\
\hline V33 & 98 & 184952 & V102 & 100 & 162564 & V168 & 0 & 124934 & V252 & 98 & 187128 & NV324 & 68 & 154689 & NV384 & 93 & 46225 \\
\hline V34 & 99 & 103189 & V103 & 98 & 195140 & V169 & 99 & 285765 & $V 253$ & 99 & 209800 & NV325 & 80 & 163821 & 85 & 99 & 35533 \\
\hline V35 & 99 & 309218 & V104 & 99 & 92705 & V184 & 100 & 134524 & V254 & 99 & 214031 & NV326 & 98 & 268304 & 86 & 98 & 218806 \\
\hline V38 & 98 & 52122 & V105 & 98 & 111797 & V185 & 99 & 320506 & V258 & 99 & 253920 & NV327 & 14 & 235193 & NV387 & 96 & 190420 \\
\hline V39 & 99 & 76656 & V106 & 99 & 202932 & V186 & 94 & 200024 & V259 & 99 & 177256 & NV328 & 69 & 129813 & NV388 & 0 & 205940 \\
\hline V40 & 99 & 150793 & V107 & 100 & 147114 & V187 & 91 & 107554 & V261 & 98 & 324469 & NV329 & 51 & 235596 & NV389 & 49 & 169916 \\
\hline V41 & 72 & 145915 & V108 & 99 & 178628 & V188 & 95 & 160942 & V263 & 100 & 243242 & NV330 & 64 & 271511 & 90 & 100 & 262766 \\
\hline V42 & 88 & 177086 & V109 & 99 & 174888 & V189 & 99 & 175684 & V264 & 98 & 157202 & NV331 & 69 & 244602 & NV391 & 99 & 46483 \\
\hline V43 & 99 & 227377 & V110 & 100 & 164532 & V190 & 99 & 243006 & V265 & 98 & 192064 & NV332 & 84 & 137605 & 93 & 99 & 36249 \\
\hline V44 & 100 & 78476 & V111 & 95 & 193829 & V191 & 99 & 301765 & V266 & 85 & 206486 & NV333 & 62 & 192856 & NV394 & 95 & 81813 \\
\hline V45 & 98 & 219445 & V112 & 78 & 158996 & V192 & 100 & 319396 & V267 & 99 & 234518 & NV334 & 88 & 183248 & NV395 & 88 & 177086 \\
\hline V46 & 99 & 249013 & V113 & 99 & 130486 & V193 & 99 & 318939 & V268 & 99 & 243048 & NV335 & 99 & 362118 & NV397 & 94 & 244021 \\
\hline V47 & 99 & 281501 & V114 & 97 & 159704 & V194 & 99 & 128211 & V270 & 98 & 164847 & NV336 & 96 & 106751 & NV398 & 96 & 267455 \\
\hline V48 & 98 & 158259 & V115 & 99 & 83123 & V195 & 81 & 274973 & V271 & 98 & 165504 & NV337 & 97 & 118163 & NV399 & 98 & 165850 \\
\hline V49 & 99 & 36832 & V116 & 56 & 204728 & V197 & 79 & 126226 & V272 & 99 & 248016 & NV338 & 98 & 136414 & NV400 & 99 & 129220 \\
\hline V50 & 99 & 214772 & V117 & 75 & 179957 & V198 & 55 & 145299 & V273 & 99 & 223751 & NV339 & 99 & 169580 & NV401 & 98 & 130105 \\
\hline V51 & 99 & 278945 & V118 & 96 & 160390 & V199 & 87 & 194465 & V274 & 77 & 305738 & NV340 & 97 & 216161 & NV402 & 87 & 52179 \\
\hline V52 & 53 & 205670 & V119 & 91 & 140617 & V200 & 75 & 243370 & V275 & 94 & 214740 & NV341 & 94 & 191217 & NV403 & 0 & 229293 \\
\hline V53 & 99 & 56123 & V120 & 99 & 110236 & V201 & 99 & 369578 & V276 & 99 & 101192 & NV342 & 99 & 199357 & NV404 & 99 & 244779 \\
\hline V54 & 98 & 352756 & V121 & 97 & 129294 & V203 & 100 & 372023 & V277 & 99 & 217316 & NV343 & 99 & 174660 & NV405 & 88 & 252122 \\
\hline V56 & 98 & 38620 & V122 & 99 & 105971 & V204 & 99 & 45537 & V280 & 99 & 300999 & NV344 & 99 & 271739 & NV406 & 100 & 292866 \\
\hline V57 & 100 & 48194 & V123 & 100 & 44090 & V205 & 96 & 302520 & V285 & 99 & 74726 & NV345 & 87 & 174082 & NV407 & 88 & 309849 \\
\hline V58 & 96 & 284348 & V124 & 99 & 26731 & V206 & 97 & 57769 & V288 & 99 & 289087 & NV346 & 90 & 202477 & NV408 & 50 & 234623 \\
\hline V59 & 96 & 171211 & V125 & 99 & 14416 & V207 & 98 & 297708 & V289 & 99 & 324714 & NV347 & 79 & 211972 & NV409 & 73 & 322122 \\
\hline V60 & 99 & 110456 & V126 & 99 & 15927 & V208 & 97 & 244408 & V291 & 98 & 97939 & NV349 & 88 & 212595 & NV410 & 0 & 180157 \\
\hline V61 & 100 & 216835 & V127 & 99 & 193936 & V209 & 99 & 297731 & V292 & 98 & 129991 & NV350 & 84 & 149574 & & & \\
\hline V62 & 99 & 208493 & V128 & 100 & 162142 & V210 & 15 & 58394 & V293 & 99 & 85152 & NV351 & 84 & 215235 & & & \\
\hline V64 & 99 & 54131 & V129 & 19 & 185687 & V211 & 90 & 298164 & NV294 & 97 & 265591 & NV352 & 99 & 183506 & & & \\
\hline V65 & 0 & 50869 & V131 & 100 & 173358 & V212 & 99 & 293439 & NV295 & 99 & 340398 & NV353 & 72 & 208251 & & & \\
\hline
\end{tabular}

Using the $1.0 \mathrm{~m}$ Swope Telescope, Kaluzny et al. (2004) measured light curves of $\sim 400$ variable stars in $\omega$ Cen, 117 of which were new identifications. We cross-checked our propermotion catalog with their own, and found a total of 338 variable stars in common, which had both $V$ and $B$ measurements in our catalog. In particular, there were 90 variable stars for which Kaluzny et al. (2004) did not provide $B$ and/or $V$ magnitudes. Our proper-motion catalog was also useful to locate these stars in the color-magnitude diagram. The position of the Kaluzny et al. (2004) variables in our CMD, as well as their membership probability, is shown in the left panel of Fig. 14. Depending on our measured membership probability, we divide the Kaluzny et al. (2004) sample into three categories: (1) $P_{\mu}<30 \%$ (red squares); (2) $30 \% \leq P_{\mu}<80 \%$ (green triangles); and (3) $P_{\mu} \geq 80 \%$ (blue circles). Of the 117 new variable identifications, 112 are in common with our catalog. Of these 112 stars, 15 have $P_{\mu}<30 \%$, and therefore these are probably not cluster members. On the other hand, 19 of these new variables have $30 \% \leq$ $P_{\mu}<80 \%$ and their membership remains uncertain. The remaining 78 stars $\left(P_{\mu} \geq 80 \%\right)$ are almost certainly $\omega$ Cen members. In Table 3, we report the membership probability values for all Kaluzny et al. (2004) variable stars identified in our catalog. 

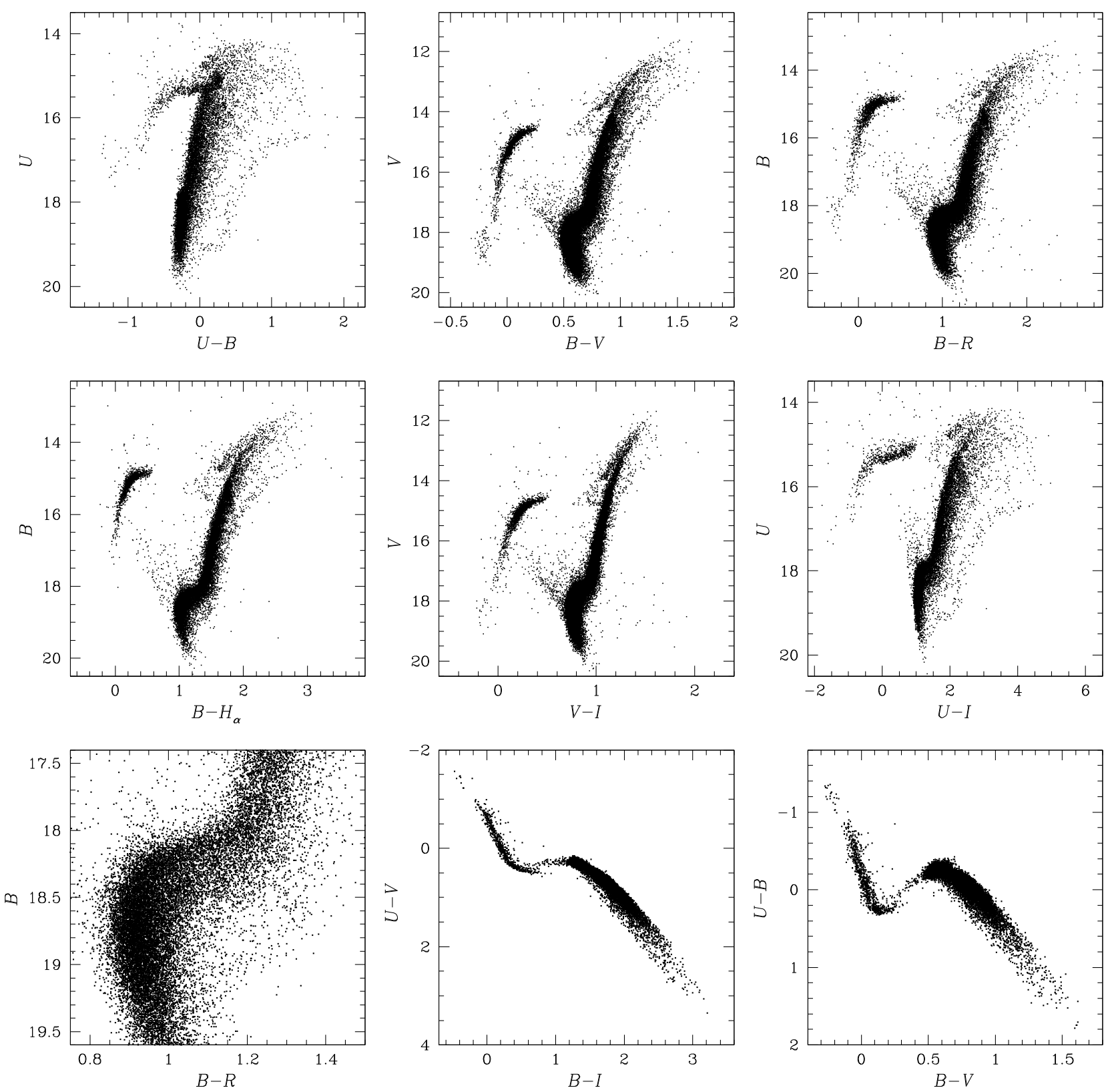

Fig. 15. Top: membership probability selected $\left(P_{\mu}>90 \%\right) \omega$ Cen CMDs. From left to right $U$ vs. $(U-B), V$ vs. $(B-V)$, and $B$ vs. $(B-R)$. Plotted stars have photometric errors going from 0.02 mag for the brighter to 0.05 for the faintest ones. Middle: same star selection criteria. From left to right: $B$ vs. $\left(B-H_{\alpha}\right), V$ vs. $(V-I)$, and $U$ vs. $(U-I)$. Bottom: the $B$ vs. $(B-R)$ CMD, zoomed in the SGB region, is shown on the left. The two plots on the right show two color-color diagrams obtained from different filters.

Table 4. Membership probability of XMM-Newton X-ray counterparts candidates. The IDs refer to Kaluzny et al. work.

\begin{tabular}{lclc}
\hline \hline $\mathrm{ID}_{\mathrm{K}}$ & $P_{\mu}$ & $\mathrm{ID}_{\mathrm{K}}$ & $P_{\mu}$ \\
\hline NV367 & 0 & $\mathrm{NV376}$ & 0 \\
NV375 & 0 & $\mathrm{NV} 377$ & 0 \\
V167 & 23 & $\mathrm{NV} 369$ & 94 \\
V223 & 0 & $\mathrm{NV} 383$ & 99 \\
NV378 & 31 & & \\
\hline
\end{tabular}

New Kaluzny et al. (2004) identifications have their IDs starting with " $N$ ".

The Kaluzny et al. (2004) variable star catalog was crosschecked with the X-ray sources detected by the XMM-Newton analysis presented by Gendre et al. (2003). For the 9 stars in common (see also Table 2 of Kaluzny et al. 2004), we can now provide (see Table 4) a membership probability based on our proper-motion analysis. A quick glance at Table 4 allows us to infer that only NV383 and NV369 are very likely cluster members, whereas the remaining 7 stars are most probably field population.

Using the $1.0 \mathrm{~m}$ Telescope of the Australian National University, Weldrake et al. (2007) detected a total of 187 variable stars covering a wide area around $\omega$ Cen. These stars were matched with the Kaluzny et al. (2004) catalog, and 81 variable stars were found to be new discoveries. A cross-check of our catalog with that of Weldrake et al. (2007) provided 102 variable stars in common. Their location in the $V$ versus $V-I$ CMD is shown in the right panel of Fig. 14. As completed for the 
Table 5. Membership probability for the Weldrake et al. (2007) variable star catalog. $\mathrm{ID}_{\mathrm{W}}$ are Weldrake et al. identification labels, $\mathrm{ID}_{\mathrm{tw}}$ come from this work. The symbol (*) marks new Weldrake et al. identifications.

\begin{tabular}{|c|c|c|c|c|c|c|c|c|c|c|c|c|c|c|c|c|c|}
\hline$\overline{\mathrm{ID}_{\mathrm{W}}}$ & 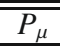 & $\overline{I I D_{t w}}$ & $\bar{c} \mathrm{ID}_{\mathrm{W}}$ & $\overline{\overline{P P_{\mu}}}$ & $\overline{\mathrm{ID}_{\mathrm{tw}}}$ & $\overline{\mathrm{ID}_{\mathrm{W}}}$ & 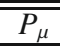 & $\bar{c} \mathrm{ID}_{\mathrm{tw}}$ & $\overline{\mathrm{ID}_{\mathrm{W}}}$ & 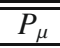 & $\overline{\mathrm{ID}_{\mathrm{tw}}}$ & $\overline{\mathrm{ID}_{\mathrm{W}}}$ & 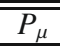 & $\overline{\mathrm{ID}_{\mathrm{tw}}}$ & $\mathrm{ID}_{\mathrm{W}}$ & 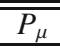 & $\overline{\mathrm{ID}_{\mathrm{tw}}}$ \\
\hline V31* & 0 & 234574 & V48 & 100 & 360819 & V102 & 15 & 58394 & V119 & 51 & 126839 & V142 & 42 & 208115 & V160* & 73 & 292364 \\
\hline V32 & 99 & 236315 & V59* & 0 & 23863 & V103 & 99 & 54131 & V120 & 68 & 154689 & V143 & 98 & 201164 & V161 & 73 & 322 \\
\hline V33 & 100 & 205260 & V60 & 48 & 40 & V104 & 93 & 462 & V121 & 53 & $15 \mathrm{c}$ & V144* & 100 & 24 & V162 & 99 & 94 \\
\hline V34 & 99 & 239985 & V61 & 100 & 48 & V105 & 99 & 83 & V122 & 100 & 162 & V145* & 99 & 270 & V163 & 93 & 174 \\
\hline V35 & 56 & 214 & V62 & 97 & 42 & V106 & 100 & 78476 & 3 & 99 & 15 & V146 & 100 & 42 & 64 & 99 & 26 \\
\hline V36 & 98 & 267 & V63 & 99 & 86 & V107 & 99 & 10 & V124 & 75 & 13 & 47 & 99 & 24 & 165 & 99 & 506 \\
\hline V37 & 0 & 243 & V64 & 99 & 84 & 88 & 48 & 92 & V & 0 & 67 & V148 & 77 & 30 & 66 & 31 & 36 \\
\hline V38 & 99 & 27 & V65 & 99 & 12 & V & 99 & 1 & V126* & 0 & 0 & 50 & 50 & 30 & 67 & 100 & 39 \\
\hline V39 & 100 & 66 & V66 & 98 & 7 & V & 99 & 1 & 3 & 94 & 61 & V & 2 & 2 & V & 64 & 75 \\
\hline V40 & 99 & 289620 & V67 & 98 & 9 & 111 & 99 & 1 & + & 53 & 00 & V152 & 100 & 29 & V169 & 100 & 63 \\
\hline V41 & 99 & 39 & V68* & 99 & 12 & V112 & 86 & 4 & 35 & 85 & 26 & V153 & 65 & 29 & V170 & 99 & 882 \\
\hline V42 & 99 & 285765 & V69* & 98 & 66 & V113 & 98 & 02 & 36 & 50 & 228136 & V154 & 98 & 287 & V171 & 98 & 352756 \\
\hline V43 & 99 & 324714 & V70 & 100 & 64 & V114 & 55 & 77 & 37 & 42 & 225221 & V155 & 64 & 28 & V172 & 96 & 358729 \\
\hline V44* & 93 & 28996 & V71* & 88 & 8 & 15 & 57 & 23 & 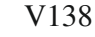 & 96 & 61 & 56 & 97 & 40 & V173 & 98 & 445 \\
\hline V45 & 99 & 6247 & V72* & 73 & 191 & V116 & 60 & 76 & 9 & 56 & 28 & V157* & 96 & 27 & V174 & 99 & 013 \\
\hline V46* & 96 & 0258 & V81* & 96 & 606 & 17 & 97 & 12 & 0 & 57 & 22 & V158 & 98 & 27 & V176 & 98 & 292312 \\
\hline V47 & 99 & 352935 & V101 & 99 & 36832 & V118 & 35 & 151268 & V141 & 51 & 218172 & V159* & 93 & 287079 & V178 & 36 & 309751 \\
\hline
\end{tabular}

Kaluzny et al. (2004) sample, different symbols mark different membership probability ranges, and Table 5 reports our membership probability for the Weldrake et al. (2007) variable catalog. Of the 81 Weldrake et al. (2007) new variable stars, 16 have counterparts in our proper-motion catalog. Four of these new variables are clearly not cluster members. These field objects include a detached eclipsing binary (V59), and three long-period variables (V31, V125, and V126).

\section{Summary}

We have applied the photometric and astrometric technique developed by Anderson et al. (2006, Paper I) to the most puzzling globular cluster of the Milky Way: $\omega$ Centauri.

Based on CCD observations taken with only four years of temporal base-line, our measurements provide accurate proper motions to $B \sim 20$, four magnitudes deeper than the photographic catalog of vL00. We have minimized the skyconcentration effects in our photometry. We provide a membership probability for all stars. Our catalog contains almost 360000 stars with measured proper motion, and covers a wide area $\left(\sim 33 \times 33 \operatorname{arcmin}^{2}\right)$ around the cluster center. In Fig. 15, we show a summary of our photometric catalog: we plot several $\omega$ Cen CMDs, derived with all the available filters and different color-baselines (top and middle rows). Plotted stars have a membership probability of $P_{\mu}>90 \%$. Photometric errors range from $0.02 \mathrm{mag}$ for brighter stars to $0.05 \mathrm{mag}$ for the fainter ones. In the bottom panels of Fig. 15, we show the SGB region of $\omega$ Cen in the $B$ versus $B-R$ CMD on the left, and two color-color diagrams with different colors on the right.

The high precision of our astrometry and multi-band photometry once again emphasizes the importance of accurate representation of the PSF across the entire field-of view, especially for wide-field imagers, exemplified by the concept of empirical PSF (Paper I).

The primary aim of this work is, of course, to provide widefield membership probability measurements for spectroscopic follow-up studies, down to the turn-off region of the cluster. However, the high quality of our photometric and astrometric measurements also provide a crucial observational constraint of the multiple $\omega$ Cen sub-populations. Due to our proper-motionselected RGB sub-populations, we can confirm that the metalpoor, metal-intermediate, and metal-rich components have the same proper motions which is that of $\omega$ Cen, within our measurements uncertainties.

We finally provide membership probability determinations for the Kaluzny et al. (2004) and Weldrake et al. (2007) $\omega$ Cen variable star catalogs.

\subsection{Electronic catalog}

The catalog is available at the SIMBAD on-line database ${ }^{2}$. Description of the catalog: Col. (1) contains the ID; Cols. (2) and (3) give the J2003.29 equatorial coordinates in decimal degrees; Cols. (4) and (5) provide the pixel coordinates $x$ and $y$ of the distortion-corrected reference meta-chip; Cols. (6) and (7) contain the proper-motion values along $\mu_{\alpha} \cos \delta$ in units of mas $\mathrm{yr}^{-1}$, with the corresponding rms; Cols. (8) and (9) provide the propermotion values along $\mu_{\delta}$, with the corresponding rms, in the same units. Columns from (10) to (21) give the photometric data, i.e. $U, B, V, R_{\mathrm{C}}, I_{\mathrm{C}}, \mathrm{H}_{\alpha}$ magnitudes and their errors. The last two columns give the proper-motion membership probability $P_{\mu}(1)$ for all the stars (22), and for a sub-sample of 120259 stars with the alternative membership determination $P_{\mu}(2)$ (23). In this case, if the second membership determination is not provided, we flag it with the value -1 .

If photometry in a specific band is not available, a flag equal to 99.999 is set for magnitude and error.

Table 6 contains the first lines of the on-line catalog.

Acknowledgements. A. Bellini acknowledges the support by the CA.RI.PA.RO. foundation, and the STScI under the 2008 graduate research assistantship program. I. Platais gratefully acknowledges support from the National Science Foundation through grant AST 04-06689 to Johns Hopkins University and and by NASA grant $H S T$-AR-09958.01-A, awarded by the Space Telescope Institute, which is operated by the Association of Universities for Research in Astronomy, Inc., under NASA contract NAS5-26555. We thank Floor van Leeuwen for his help in providing us a complete version of his catalog as well as stimulating discussions. We thank the anonymous referee for the careful reading of the manuscript, and for the useful comments.

\section{References}

Andersen, M. I., Freyhammer, L., \& Storm, J. 1995, Calibrating and Understanding HST and ESO Instruments, 87 Anderson, J. 1997, Ph.D. Thesis, Univ. California, Berkeley

${ }^{2}$ http://simbad.u-strasbg.fr/simbad/ 


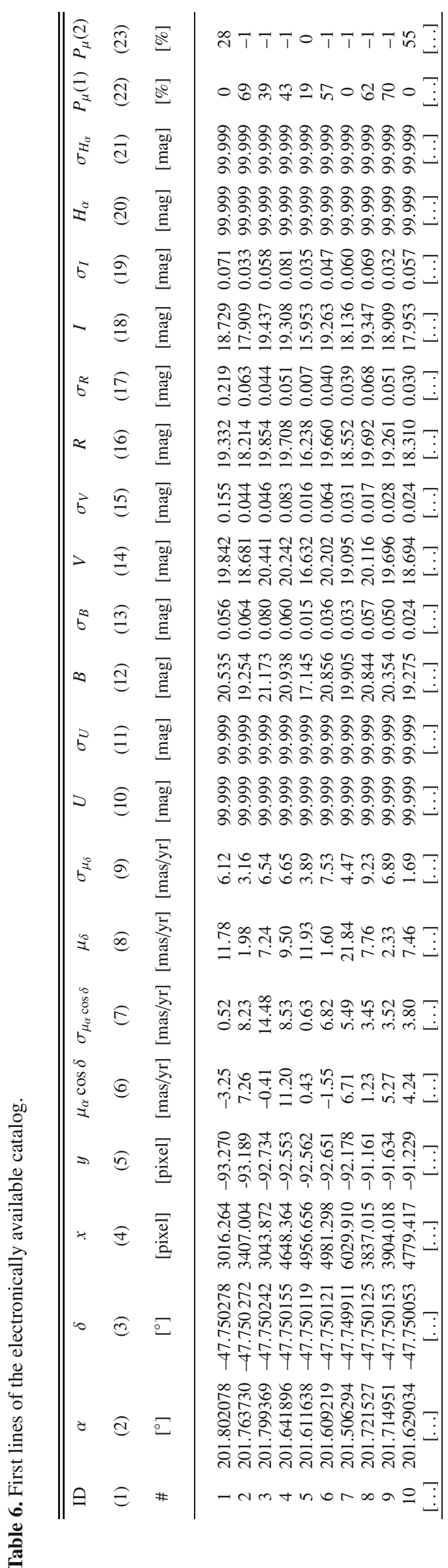

Anderson, J. 2003, in New Horizons in Globular Cluster Astronomy, ed. G. Piotto, G. Meylan, D. Djorgovski, \& M. Riello (San Francisco: ASP), ASP Conf. Ser., 296, 125

Anderson, J., \& King, I. R. 2003, PASP, 115, 113

Anderson, J., Bedin, L. R., Piotto, G., Yadav, R. S., \& Bellini, A. 2006, A\&A, 454, 1029 (Paper I)

Anderson, J., Sarajedini, A., Bedin, L. R., et al. 2008, AJ, 135, 2055

Balaguer-Núnez, L., Tian, K. P., \& Zhao, J. L. 1998, A\&AS, 133, 387

Bedin, L. R., Piotto, G., Anderson, J., et al. 2004, ApJ, 605, L125

Bedin, L. R., Cassisi, S., Castelli, F., et al. 2005, MNRAS, 357, 1038 Bessell, M. S., \& Norris, J. 1976, ApJ, 208, 369

Butler, D., Dickens, R. J., \& Epps, E. 1978, ApJ, 225, 148

Calamida, A., Stetson, P. B., Bono, G., et al. 2005, ApJ, 634, L69

Calamida, A., Corsi, C. E., Bono, G., et al. 2008, [arXiv:0801.0693]

Cassisi, S., Schlattl, H., Salaris, M., \& Weiss, A. 2003, ApJ, 582, L43

Del Principe, M., Piersimoni, A. M., Storm, J., et al. 2006, ApJ, 652, 362

Ferraro, F. R., Bellazzini, M., \& Pancino, E. 2002, ApJ, 573, L95

Ferraro, F. R., Beccari, G., Rood, R. T., et al. 2004, ApJ, 603, 127

Freeman, K. C., \& Rodgers, A. W. 1975, ApJ, 201, L71

Gendre, B., Barret, D., \& Webb, N. A. 2003, A\&A, 400, 521

Harris, W. E. 1996, AJ, 112, 1487

Hilker, M., Kayser, A., Richtler, T., \& Willemsen, P. 2004, A\&A, 422, L9

Hughes, J., Wallerstein, G., van Leeuwen, F. \& Hilker, M. 2004, AJ, 127, 980

Johnson, C. I., Pilachowski, C. A., Simmerer, J., \& Schwenk, D. 2008, ApJ, accepted [arXiv:0804.2607]

Kaluzny, J., Olech, A., Thompson, I. B., et al. 2004, A\&A, 424, 1101

Koch, A., Odenkirchen, M., Grebel, E. K., \& Caldwell, J. A. R. 2004, Astron. Nachr., 325, 299

Lee, Y.-W., Joo, J.-M., Sohn, Y.-J., et al. 1999, Nature, 402, 55

Manfroid, J., \& Selman, F. 2001, The Messenger, 104, 16

Merritt, D., Meylan, G., \& Mayor, M. 1997, AJ, 114, 1074

Meylan, G., Mayor, M., Duquennoy, A., \& Dubath, P. 1995, A\&A, 303, 761

Momany, Y., Cassisi, S., Piotto, G., et al. 2003, A\&A, 407, 303

Monet, D. G., Dahn, C. C., Vrba, F. J., et al. 1992, AJ, 103, 638

Norris, J., \& Bessell, M. S. 1975, ApJ, 201, L75

Norris, J., \& Bessell, M. S. 1977, ApJ, 211, L91

Norris, J. E. 2004, ApJ, 612, 25

Norris, J. E., \& Da Costa, G. S. 1995, ApJ, 441, L81

Norris, J. E., Freeman, K. C., \& Mighell, K. J. 1996, ApJ, 462, 241

Pancino, E., Ferraro, F. R., Bellazzini, M., Piotto, G., \& Zoccali, M. 2000, ApJ, 534, L83

Pancino, E., Galfo, A., Ferraro, F. R., \& Bellazzini, M. 2007, ApJ, 661, L155

Piotto, G., Villanova, S., Bedin, L. R., et al. 2005, ApJ, 621, 777

Platais, I., Wyse, R. F. G., Hebb, L., Lee, Y.-W., \& Rey, S.-C. 2003, ApJ, 591, L127

Rey, S.-C., Lee, Y.-W., Ree, C. H., et al. 2004, AJ, 127, 958

Sanders, W. L. 1971, A\&A, 14, 226

Sarajedini, A., Barker, M. K., Geisler, D., et al. 2007, AJ, 133, 1658

Selman, F. J. 2001, Determining a zero-point variation map for the WFI, http://www.1s.eso.org/lasilla/Telescopes/2p2T/E2p2M/WFI/ zeropoints

Selman, F. J., \& Melnick, J. 2005, A\&A, 443, 851

Sirianni, M., Jee, M. J., Benítez, N., et al. 2005, PASP, 117, 1049

Sollima, A., Pancino, E., Ferraro, F. R., et al. 2005, ApJ, 634, 332

Stanford, L. M., Da Costa, G. S., Norris, J. E., \& Cannon R. D. 2006, ApJ, 647, 1075

Stetson, P. B. 2000, PASP, 112, 925

Stetson, P. B. 2005, PASP, 117, 563

Suntzeff, N. B., \& Kraft, R. P. 1996, AJ, 111, 1913

Tian, K.-P., Zhao, J.-L., Shao, Z.-Y., \& Stetson, P. B. 1998, A\&AS, 131, 89

van de Ven, G., van den Bosch, R. C. E., Verolme, E. K., \& de Zeeuw, P. T. 2006, A\&A, 445, 513

van Leeuwen, F., Le Poole, R. S., Reijns, R. A., Freeman, K. C., \& de Zeeuw, P. T. 2000, A\&A, 360, 472 (vL00)

Vasilevskis, S., Klemola, A., \& Preston, G. 1958, AJ, 63, 387

Villanova, S., Piotto, G., King, I. R., et al. 2007, ApJ, 663, 296

Weldrake, D. T. F., Sackett, P. D., \& Bridges, T. J. 2007, AJ, 133, 1447

Yadav, R. K. S., Bedin, L. R., Piotto, G., et al. 2008, A\&A, 484, 609 (Paper II)

Zacharias, N., Urban, S. E., Zacharias, M. I., et al. 2004, AJ, 127, 3043

Zhao, J. L., \& He, Y. P. 1990, A\&A, 237, 54 